\title{
High order semi-implicit weighted compact nonlinear scheme for the all-Mach isentropic Euler system
}

\author{
Yanqun Jiang ${ }^{1 * \dagger}$, Xun Chen $^{1 \dagger}$, Xu Zhang $^{1 \dagger}$, Tao Xiong $^{2 \dagger}$ and Shuguang Zhou ${ }^{3 \dagger}$
}

\author{
${ }^{*}$ Correspondence: \\ jyq2005@mail.ustc.edu.cn \\ †Yanqun Jiang, Xun Chen, Xu \\ Zhang, Tao Xiong and Shuguang \\ Zhou contributed equally to this \\ work. \\ 'Department of Mathematics, \\ Southwest University of Science \\ and Technology, 621010 Mianyang, \\ China \\ Full list of author information is \\ available at the end of the article
}

\begin{abstract}
The computation of compressible flows at all Mach numbers is a very challenging problem. An efficient numerical method for solving this problem needs to have shock-capturing capability in the high Mach number regime, while it can deal with stiffness and accuracy in the low Mach number regime. This paper designs a high order semi-implicit weighted compact nonlinear scheme (WCNS) for the all-Mach isentropic Euler system of compressible gas dynamics. To avoid severe Courant-Friedrichs-Levy $(\mathrm{CFL})$ restrictions for low Mach flows, the nonlinear fluxes in the Euler equations are split into stiff and non-stiff components. A third-order implicit-explicit (IMEX) method is used for the time discretization of the split components and a fifth-order WCNS is used for the spatial discretization of flux derivatives. The high order IMEX method is asymptotic preserving and asymptotically accurate in the zero Mach number limit. One- and two-dimensional numerical examples in both compressible and incompressible regimes are given to demonstrate the advantages of the designed IMEX WCNS.
\end{abstract}

Keywords: High order scheme, IMEX time discretization, WCNS, Asymptotic-preserving property, Low Mach number, Isentropic Euler equations

\section{Introduction}

Low-Mach number flows in fluid dynamics that are slow compared with the speed of sound can be described by the following scaled isentropic Euler equations.

$$
\left\{\begin{aligned}
\rho_{t}+\nabla \cdot(\rho \mathbf{u}) & =0 \\
(\rho \mathbf{u})_{t}+\nabla \cdot(\rho \mathbf{u} \otimes \mathbf{u})+\frac{1}{\varepsilon^{2}} \nabla p & =0
\end{aligned}\right.
$$

where $\rho(\mathbf{x}, t)>0, \mathbf{u}(\mathbf{x}, t)=(u(\mathbf{x}, t), v(\mathbf{x}, t))$ and $\mathbf{q}=\rho \mathbf{u}(\mathbf{x}, t)$ are the density, velocity and momentum of the fluid, respectively, and $\varepsilon>0$ is the scaled Mach number that is a measure of compressibility of the fluid. For example, $\varepsilon=1$ corresponds to the fully compressible regime. The operators $\nabla, \nabla \cdot$ and $\otimes$ are the gradient, divergence and tensor product operators, respectively. The pressure $p$ in Eq. (1) is given by the equation of state

$$
p(\rho)=\Lambda \rho^{\gamma}
$$

(C) The Author(s). 2020 Open Access This article is licensed under a Creative Commons Attribution 4.0 International License which permits use, sharing, adaptation, distribution and reproduction in any medium or format, as long as you give appropriate credit to the original author(s) and the source, provide a link to the Creative Commons licence, and indicate if changes were made. The images or other third party material in this article are included in the article's Creative Commons licence, unless indicated otherwise in a credit line to the material. If material is not included in the article's Creative Commons licence and your intended use is not permitted by statutory regulation or exceeds the permitted use, you will need to obtain permission directly from the copyright holder. To view a copy of this licence, visit http://creativecommons.org/licenses/by/4.0/. 
where $\Lambda>0$ and $\gamma \geq 1$ are constants. Equation (1) are hyperbolic with wave speeds in direction $\mathbf{n}$

$$
\lambda_{1,3}=\mathbf{u} \cdot \mathbf{n} \pm c, \lambda_{2}=\mathbf{u} \cdot \mathbf{n},
$$

and the sound speed $c=\sqrt{p^{\prime}(\rho)} / \varepsilon$.

Klainerman and Majda [1, 2] proved that the solution of Eq. (1) converges to the incompressible counterpart when $\varepsilon \sim 0$. The asymptotic nature of the solution to Eq. (1) can be studied by plugging-in the ansatz

$$
f(\mathbf{x}, t)=f_{0}(\mathbf{x}, t)+\varepsilon^{2} f_{2}(\mathbf{x}, t)+O\left(\varepsilon^{3}\right),
$$

for variables $\rho, p$ and $\mathbf{u}$. After performing a scale analysis, we obtain an incompressible Euler system for the velocity

$$
\left\{\begin{aligned}
\left(\mathbf{u}_{0}\right)_{t}+\nabla \cdot\left(\mathbf{u}_{0} \otimes \mathbf{u}_{0}\right)+\frac{\nabla p_{2}}{\rho_{0}} & =0, \\
\nabla \cdot \mathbf{u}_{0} & =0,
\end{aligned}\right.
$$

based on well-prepared initial data, i.e.,

$$
\rho(\mathbf{x}, 0)=\text { const }+O\left(\varepsilon^{2}\right), \nabla \cdot \mathbf{u}(\mathbf{x}, 0)=O(\varepsilon),
$$

and appropriate boundary conditions [3], e.g., periodic, wall or homogeneous Neumann boundary conditions. Taking the divergence of the first equation in (5) and using the incompressibility, we obtain

$$
\triangle p_{2}=-\nabla \cdot\left(\nabla \cdot\left(\rho_{0} \mathbf{u}_{0} \otimes \mathbf{u}_{0}\right)\right) .
$$

In low Mach number flows, the challenge faced by standard hyperbolic numerical methods for Eq. (1) is that the system becomes stiff. The stiffness is caused by the stringent Courant-Friedrichs-Levy (CFL) restriction [3-6], i.e. $\Delta t \leq \Delta x / \max _{i}\left|\lambda_{i}\right|=\mathcal{O}(\varepsilon \Delta x)$ with the spatial and temporal grid sizes $\Delta x$ and $\Delta t$. The restriction results in an increasingly large computational time for $\varepsilon \rightarrow 0$. Moreover, the strong stability requirement for $\varepsilon \rightarrow 0$ results in the excessive numerical viscosity of the standard hyperbolic solvers that is proportional to $\max _{i}\left|\lambda_{i}\right|=\mathcal{O}\left(\varepsilon^{-1}\right)$. This makes the standard hyperbolic solvers suffer from severe loss of accuracy due to the creation of spurious waves. Therefore, the stability and accuracy of the standard hyperbolic numerical methods highly depend on $\varepsilon$ [4].

In the literature, some all-speed schemes [3-16] have been derived for the compressible-incompressible limit problems based on the idea of the asymptoticpreserving (AP) methodology introduced by Jin $[17,18]$ for relaxation systems. The AP all-speed schemes are uniformly consistent with the low-Mach number limit and are uniformly stable. Although a robust AP scheme should allow both explicit and implicit time-marching techniques, the explicit strategies suffer from the CFL stability conditions and thus affect the efficiency of the method for small Mach numbers. The implicit strategies avoid the CFL restriction, but they need to solve highly nonlinear equations and add an excessive numerical dissipation to the slow wave, degrading the accuracy of the method [3]. To overcome the drawbacks, the semi-implicit or implicit-explicit (IMEX) time discretization strategies are widely applied based on the acoustic/advection splitting of the Euler equations into the stiff/non-stiff parts (or fast and slow fluxes) $[3-5,10]$. These strategies treat with the stiff part implicitly and the non-stiff part explicitly to solve the isentropic Euler equations effectively and efficiently. The IMEX time discretization combined with the shock-capturing schemes, e.g., the first-order local 
Lax-Friedrichs (or Rusanov) scheme [4], the second-order finite volume method [3, 19], the arbitrary high order accurate discontinuous Galerkin method [20] and the fifth-order finite difference weighted essentially non-oscillatory (WENO) scheme [10], has shown its good performances in compressible and incompressible regimes.

Recently a class of weighted compact nonlinear schemes (WCNS's) as high order accurate shock-capturing schemes have been widely adopted to investigate compressible flows with shock waves and discontinuities [21-27]. The WCNS's are first proposed by Deng and Zhang [21] based on the cell-centered compact scheme of Lele [28] and are developed in [22-24,29]. The expression of the classical cell-centered compact scheme is that a linear combination of the unknown approximations of the first derivative of a function at the grid points in a stencil is equal to another linear combination of the function itself at the grid points in the same stencil. Compared to noncompact schemes, e.g., the classical WENO scheme [30], the stencil can be more compact in the compact scheme for the same order of accuracy [22]. WCNS's that integrate WENO interpolation into the cell-centered compact scheme for the first derivative of a function are variants of the WENO schemes and have the good performance in terms of accuracy, robustness and efficiency for compressible flows. Compared to the classical WENO schemes, the WCNS's feature a better spectral resolution at the same order of accuracy [21, 22]. They are more flexible in the choice of flux splitting methods, e.g., Roe's flux difference splitting method, van Leer's flux vector splitting and Liou's advection upstream splitting method, and flux derivatives can be computed by different explicit or implicit compact finite difference methods [23, 29]. In this work, in view of the good performance of the IMEX time discretization method and the WCNS's, we design a high order IMEX WCNS for the all-Mach isentropic Euler system based on the acoustic/advection splitting strategy [10]. The stiff part governing fast acoustic and the non-stiff part modeling slow nonlinear advection effects are solved by the high order implicit and explicit time discretization methods, respectively. In the zero Mach number limit, the high order IMEX scheme has the AP property and is asymptotically stable under a convective CFL condition independent of the Mach number $\varepsilon$. Oneand two-dimensional numerical examples are given to demonstrate good performance of the designed IMEX WCNS in both compressible and incompressible regimes.

\section{WCNS method}

In this section, a fifth-order WCNS is considered for the spatial discretization of flux derivatives. The WCNS procedure consists of three components, i.e., midpoint-to-node differencing, flux evaluation at the midpoint and node-to-midpoint weighted averaging interpolation of fluxes. Here, we briefly describe the framework of WCNS discretization for the first derivative, $f_{x}$, in the one-dimensional scalar case $u_{t}+f(u)_{x}=0$. For simplicity, we take a uniform grid defined by $x_{i}=i \Delta x=i h, i=0, \ldots, N$, where $h$ is the uniform grid spacing and $N$ is the number of cells.

The classical cell-centered compact scheme for the first derivatives [28] has no dissipation, but the dispersive errors dominate the scheme, which may destroy the numerical solutions. A class of hybrid cell-node and cell-edge WCNS's proposed in [29] overcome the disadvantages and are written as

$$
f_{i}^{\prime}+\sum_{k=1}^{q} \varrho_{k}\left(f_{i+k}^{\prime}+f_{i-k}^{\prime}\right)=\frac{1}{h} \sum_{m=1}^{r} \zeta_{m}\left(f_{i+(m-1 / 2)}+f_{i-(m-1 / 2)}\right)+\frac{1}{h} \sum_{l=1}^{w} \eta_{l}\left(f_{i+l}+f_{i-l}\right),
$$


which introduce dissipation by approximating the cell-edge fluxes $f_{i \pm(m-1 / 2)}$ based on nonlinear weighted averaging interpolation of fluxes. The coefficients $\varrho_{k}, \eta_{l}$ and $\zeta_{m}$ in Eq. (8) can be acquired by matching the Taylor series coefficients of various orders. If the coefficients $\varrho_{k}=0$, a class of explicit WCNS's can be derived. The explicit WCNS's are preferred because of their low cost and simplicity for parallelization and vectorization, compared to the implicit WCNS's with a certain coefficient $\varrho_{k^{*}} \neq 0$. Here, we consider one of explicit WCNS's and use a sixth-order explicit central-type scheme obtained from Eq. (8) to approximate $f_{x}$, i.e.,

$$
f_{i}^{\prime}=\frac{64}{45 h}\left(\hat{f}_{i+1 / 2}-\hat{f}_{i-1 / 2}\right)-\frac{2}{9 h}\left(f_{i+1}-f_{i-1}\right)+\frac{1}{180 h}\left(f_{i+2}-f_{i-2}\right) .
$$

By the Taylor series expansion of (9), we obtain

$$
f_{i}^{\prime}=\left(f_{x}\right)_{i}-\frac{1}{5040} h^{6}\left(\frac{\partial^{7} f}{\partial x^{7}}\right)_{i}+O\left(h^{8}\right)+O\left(h^{r}\right),
$$

where the truncation term $O\left(h^{r}\right)$ comes from the numerical approximations of the celledge fluxes $f_{i \pm 1 / 2}$, denoted by $\hat{f}_{i \pm 1 / 2}$. The accuracy of the scheme (9) is $\min (6, r)$. Here, the numerical fluxes $\hat{f}_{i \pm 1 / 2}$ approximate $f_{i \pm 1 / 2}$ to the fifth order and thus a fifth-order WCNS is designed for the spatial discretization.

Various flux splitting methods [23] can be used in WCNS to introduce correct upwinding. For simplicity, we use the Lax-Friedrichs flux splitting, i.e., the flux is split into positive and negative parts:

$$
f=f^{+}+f^{-}, f^{ \pm}=\frac{1}{2}(f \pm \alpha u)
$$

where $\alpha=\max _{u}\left|f_{u}^{\prime}\right|$ is the maximum characteristic wave speed. The numerical fluxes $\hat{f}_{i \pm 1 / 2}$ at the cell centers in (9) are calculated as

$$
\hat{f}_{i \pm 1 / 2}=\hat{f}_{i \pm 1 / 2}^{+} \pm \hat{f}_{i \pm 1 / 2}^{-} .
$$

A fifth-order WENO interpolation technique is considered to obtain the positive numerical flux $\hat{f}_{i+1 / 2}^{+}$. The interpolation process of the negative numerical flux $\hat{f}_{i+1 / 2}^{-}$is symmetric with respect to $x_{i+1 / 2}$ and will not be shown here. For simplicity, the "+" sign in the superscript is removed.

The numerical flux $\hat{f}_{i+1 / 2}$ on a cell center can be interpolated locally from the values on a five-point upwind-biased stencil $S=\left\{x_{i \pm 2}, x_{i \pm 1}, x_{i}\right\}$. The stencil $S$ can be divided into three sub-stencils $S_{k}=\left\{x_{i+k-2}, x_{i+k-1}, x_{i+k}\right\}, k=0,1,2$. On each sub-stencil a thirdorder linear interpolation of $f$ at cell center $x_{i+1 / 2}$ can be derived, i.e.,

$$
\begin{aligned}
\hat{f}_{i+1 / 2}^{0} & =\frac{1}{8}\left(3 f_{i-2}-10 f_{i-1}+15 f_{i}\right), \\
\hat{f}_{i+1 / 2}^{1} & =\frac{1}{8}\left(-f_{i-1}+6 f_{i}+3 f_{i+1}\right), \\
\hat{f}_{i+1 / 2}^{2} & =\frac{1}{8}\left(3 f_{i}+6 f_{i+1}-f_{i+2}\right),
\end{aligned}
$$

where $\hat{f}_{i+1 / 2}^{k}, k=0,1,2$, are the approximated values at cell center $x_{i+1 / 2}$ on $S_{k}, k=0,1,2$, respectively. On the stencil $S$ a fifth-order upwind-biased linear interpolation of $f$ at cell center $x_{i+1 / 2}$ can be derived, i.e.,

$$
\hat{f}_{i+1 / 2}^{u p w i n d}=\frac{1}{128}\left(3 f_{i-2}-20 f_{i-1}+90 f_{i}+60 f_{i+1}-5 f_{i+2}\right),
$$


which can also be constructed linearly from the three third-order interpolations $\hat{f}_{i+1 / 2}^{k}$ :

$$
\hat{f}_{i+1 / 2}^{\text {upwind }}=\sum_{k=0}^{2} d_{k} \hat{f}_{i+1 / 2}^{k}
$$

with the linear weights

$$
d_{0}=\frac{1}{16}, d_{1}=\frac{10}{16}, d_{2}=\frac{5}{16} .
$$

The central-type scheme (9) with the upwind-biased linear interpolation (16) or (17) will generate oscillations around shock waves and discontinuities. To capture shock waves and discontinuities, the numerical flux $\hat{f}_{i+1 / 2}$ should be computed by using the nonlinear combination of upwind-biased interpolations, i.e.,

$$
\hat{f}_{i+1 / 2}=\sum_{k=0}^{2} \omega_{k} \hat{f}_{i+1 / 2}^{k}
$$

where $\omega_{k}, k=0,1,2$, are the upwind-biased nonlinear weights that are associated with the relative smoothness of $f$ on each sub-stencil. In smooth areas the nonlinear weights $\omega_{k}$ should be close to the linear weights $d_{k}$ to achieve the designed order and be close to zero near discontinuities to suppress spurious numerical oscillations near discontinuities.

There are several types of nonlinear weights $\omega_{k}$ in the literature, e.g., the WENO-JS [30], WENO-M [31] and WENO-Z [32-34] weights. The WENO-Z weights place a larger weight to the less smooth sub-stencil, and thus they are less dissipative than the WENOJS weights and more efficient than the WENO-M weights. Here, the WENO-Z weights $\omega_{k}$ proposed by Borges et al. [32] are chosen.

$$
\omega_{k}=\frac{\alpha_{k}}{\sum_{k=0}^{2} \alpha_{k}}, \quad \alpha_{k}=d_{k}\left(1+\frac{\tau}{\beta_{k}+\delta}\right),
$$

where the second-order smoothness indicators $\beta_{k}, k=0,1,2$, derived by $\mathrm{Hu}$ [35] are adopted to measure the smoothness of $f$ on the sub-stencils $S_{k}$.

$$
\beta_{k}=\frac{1}{4}\left(a_{k}\right)^{2}+\frac{13}{12}\left(b_{k}\right)^{2}-\left|a_{k} b_{k}\right|,
$$

with

$$
\begin{gathered}
a_{0}=f_{i-2}-4 f_{i-1}+3 f_{i}, \quad a_{1}=f_{i-1}-f_{i+1}, \quad a_{2}=3 f_{i}-4 f_{i+1}+f_{i+2}, \\
b_{0}=f_{i-2}-2 f_{i-1}+f_{i}, \quad b_{1}=f_{i-1}-2 f_{i}+f_{i+1}, \quad b_{2}=f_{i}-2 f_{i+1}+f_{i+2} .
\end{gathered}
$$

In (20), the global higher order smoothness indicator $\tau=\left|\beta_{0}-\beta_{2}\right|$ and the parameter $\delta$ is a small constant to avoid denominators becoming zero.

Remark 1 The value of $\delta$ in (20) will influence the convergence, accuracy and stability of the schemes. Here, a parameter-free algorithm proposed by Zheng et al. [36] is used to calculate the value of $\delta$ as follows.

$$
\delta=\frac{\beta_{\text {ave }} \beta_{\min }}{\beta_{\text {std }}}
$$

where $\beta_{\text {ave }}=\frac{1}{3} \sum_{k=0}^{2} \beta_{k}, \beta_{\text {min }}=\min \left\{\beta_{0}, \beta_{1}, \beta_{2}\right\}, \beta_{\text {std }}=\sqrt{\frac{1}{3} \sum_{k=0}^{2}\left(\beta_{\text {ave }}-\beta_{k}\right)^{2}}$. To avoid denominators becoming zero in (20) and (22), $\beta_{k}+\delta$ in (20) and $\beta_{\text {std }}$ in (22) are replaced with $\max \left(\beta_{k}+\delta\right.$, err $)$ and $\max \left(\beta_{\text {std }}\right.$, err $)$, respectively. Here, err is the square root of the smallest positive number allowed for a machine. 


\section{IMEX method}

In this section, we briefly describe the framework of IMEX time discretization designed for the numerical integration of the stiff ordinary differential equation (ODE) as follows.

$$
y_{t}=\bar{f}(y)+\bar{g}(y),
$$

where $\bar{f}$ and $\bar{g}$ are the non-stiff and stiff parts of the ODE, respectively. The IMEX time discretization treats the non-stiff part explicitly and the stiff part implicitly in order to overcome the stringent time-step restriction for stability. There are many IMEX methods designed for various problems in the literature [37-41]. Among them, the IMEX Runge-Kutta (IMEX-RK) schemes as combinations of diagonally implicit A- or L-stable RK (DIRK) methods and explicit RK methods offer a precise and robust approach to define high order AP schemes. The computational cost per time step is proportional to the number of stages in the IMEX-RK schemes and the accuracy is proportional to the time-step size and the order of accuracy.

The $\operatorname{IMEX}-\operatorname{RK} p(s, \sigma, p)$ scheme is characterized by the $s \times s$ matrices $\mathbf{A}=\left(a_{i j}\right), \tilde{\mathbf{A}}=$ $\left(\tilde{a}_{i j}\right)$, the vectors $\mathbf{b}, \tilde{\mathbf{b}} \in \mathbb{R}^{s}$, and the vectors $\mathbf{c}, \tilde{\mathbf{c}} \in \mathbb{R}^{s}$. This scheme with $s$ stages of the implicit scheme for the stiff term and $\sigma(=s+1)$ stages of the explicit scheme for the nonstiff term has $p^{\text {th }}$-order temporal accuracy. Applying this scheme to solve Eq. (23) from $t^{n}$ to $t^{n+1}$ yields

$$
\begin{aligned}
y^{(k)} & =y^{n}+\Delta t \sum_{j=1}^{k-1} \tilde{a}_{k} \bar{f}\left(y^{(j)}\right)+\Delta t \sum_{j=1}^{k} a_{k j} \bar{g}\left(y^{(j)}\right), k=1, \ldots, s, \\
y^{n+1} & =y^{n}+\Delta t \sum_{k=1}^{s} \tilde{b}_{k} \bar{f}\left(y^{(k)}\right)+\Delta t \sum_{k=1}^{s} b_{k} \bar{g}\left(y^{(k)}\right) .
\end{aligned}
$$

Definition 1 The IMEX-RKp $(s, \sigma, p)$ scheme is of type ARS, i.e., the matrices $\mathbf{A}, \tilde{\mathbf{A}}$ for the implicit and explicit schemes are described respectively as

$$
\mathbf{A}=\left(\begin{array}{cc}
0 & 0 \\
0 & A_{22}
\end{array}\right), \tilde{\mathbf{A}}=\left(\begin{array}{cc}
0 & 0 \\
\tilde{A}_{21} & \tilde{A}_{22}
\end{array}\right),
$$

with the submatrices $\left(\tilde{A}_{21}\right)_{(s-1) \times 1},\left(\tilde{A}_{22}\right)_{(s-1) \times(s-1)}$ and the invertible submatrix $\left(A_{22}\right)_{(s-1) \times(s-1)}$.

Definition 2 The IMEX-ARSp scheme is globally stiffly accurate (GSA) (see [10, 40]), i.e., the matrices $\mathbf{A}, \tilde{\mathbf{A}}$ and the vectors $\mathbf{b}^{T}, \tilde{\mathbf{b}}^{T}$ satisfy

$$
\mathbf{e}_{s}^{T} \mathbf{A}=\mathbf{b}^{T}, \quad \mathbf{e}_{s}^{T} \tilde{\mathbf{A}}=\tilde{\mathbf{b}}^{T}, \quad c_{s}=\tilde{c}_{s}=1,
$$

where $\mathbf{e}_{s}=(0, \ldots, 0,1)^{T}$. The IMEX-ARSp scheme that satisfies the condition $\mathbf{e}_{s}^{T} \mathbf{A}=\mathbf{b}^{T}$ is called stiffly accurate (SA).

Remark 2 For the GSA IMEX-ARSp scheme, $y^{n+1}=y^{(s)}$ in Eq. (25).

\section{IMEX-ARSp wCNS}

In this section, we design the IMEX-ARSp WCNS scheme for the isentropic Euler system. Here, the fifth-order WCNS described in the previous section is used for the spatial discretization and the $p^{\text {th }}$-order IMEX-ARS $p$ method is used for the time dis- 
cretization. We first split the Euler system into two parts based on the acoustic/advection splitting strategy [10] and obtain

$$
\mathbf{U}_{t}=\mathbf{F}+\mathbf{G},
$$

where $\mathbf{U}=(\rho, \mathbf{q}), \mathbf{F}=-\left(0, \nabla \cdot \frac{\mathbf{q} \otimes \mathbf{q}}{\rho}\right)$ is the non-stiff nonlinear term and $\mathbf{G}=-(\nabla$. q, $\left.\frac{1}{\varepsilon^{2}} \nabla p\right)$ is the stiff linear term.

The $p^{\text {th }}$-order IMEX-ARS $p$ scheme that consists of (24) and (25) is applied to each component of (28). The intermediate stages are described as

$$
\begin{aligned}
& \rho^{(k)}=\rho^{n}-\Delta t \sum_{j=1}^{k} a_{k j} \nabla \cdot \mathbf{q}^{(j)}, \\
& \mathbf{q}^{(k)}=\mathbf{q}^{n}-\Delta t \sum_{j=1}^{k-1} \tilde{a}_{k j}\left(\nabla \cdot \frac{\mathbf{q} \otimes \mathbf{q}}{\rho}\right)^{(j)}-\Delta t \sum_{j=1}^{k} a_{k j} \frac{1}{\varepsilon^{2}} \nabla p^{(j)},
\end{aligned}
$$

where $k=1,2, \ldots, s$, and the numerical solutions at time $t^{n+1}$ are described as

$$
\begin{aligned}
& \rho^{n+1}=\rho^{n}-\Delta t \sum_{k=1}^{s} b_{k} \nabla \cdot \mathbf{q}^{(k)}, \\
& \mathbf{q}^{n+1}=\mathbf{q}^{n}-\Delta t \sum_{k=1}^{s} \tilde{b}_{k}\left(\nabla \cdot \frac{\mathbf{q} \otimes \mathbf{q}}{\rho}\right)^{(k)}-\Delta t \sum_{k=1}^{s} b_{k} \frac{1}{\varepsilon^{2}} \nabla p^{(k)} .
\end{aligned}
$$

The intermediate stages (29) and (30) consist of two implicit steps and can be simplified by eliminating $\mathbf{q}^{(k)}$ between (29) and (30) [10], and obtain a nonlinear elliptic equation for $\rho^{(k)}$ :

$$
-\frac{\left(\Delta t a_{k k}\right)^{2}}{\varepsilon^{2}} \Delta p\left(\rho^{(k)}\right)+\rho^{(k)}=\hat{\rho}^{(k)}-\Delta t a_{k k} \nabla \cdot \hat{\mathbf{q}}^{(k)},
$$

where

$$
\begin{aligned}
\hat{\rho}^{(k)} & =\rho^{n}-\Delta t \sum_{j=1}^{k-1} a_{k j} \nabla \cdot \mathbf{q}^{(j)}, \\
\hat{\mathbf{q}}^{(k)} & =\mathbf{q}^{n}-\Delta t \sum_{j=1}^{k-1} \tilde{a}_{k j}\left(\nabla \cdot \frac{\mathbf{q} \otimes \mathbf{q}}{\rho}\right)^{(j)}-\Delta t \sum_{j=1}^{k-1} a_{k j} \frac{1}{\varepsilon^{2}} \nabla p^{(j)} .
\end{aligned}
$$

Based on (30) and (35), $\mathbf{q}^{(k)}$ can be calculated as

$$
\mathbf{q}^{(k)}=\hat{\mathbf{q}}^{(k)}-\Delta t a_{k k} \frac{1}{\varepsilon^{2}} \nabla p^{(k)}
$$

Remark 3 To update the solutions from $t^{n}$ to $t^{n+1}$ by the $p^{\text {th }}$-order IMEX-ARSp WCNS, we first solve the elliptic equation (33) to obtain $\rho^{(k)}$ and then calculate explicitly $\mathbf{q}^{(k)}$ using (36). Here, (33) is a nonlinear equation for $\rho^{(k)}$, which is solved by inexact Newtongeneralized minimum residual (Newton-GMRES) algorithms. Refer to [42, 43] for the detailed description of the algorithms. Finally, the updates $\rho^{n+1}$ and $\mathbf{q}^{n+1}$ are calculated explicitly using (31) and (32) with the intermediate values $\rho^{(k)}$ and $\mathbf{q}^{(k)}$. For the GSA IMEX-ARSp scheme, $\rho^{n+1}=\rho^{(s)}$ and $\mathbf{q}^{n+1}=\mathbf{q}^{(s)}$.

Remark 4 The spatial discretization of both convective terms in (33)-(35) and the gradient terms in (34) and (35) are solved by the fifth-order WCNS in the component-bycomponent and dimension-by-dimension forms. Here, in the interpolation process of the 
cell-edge pressures, the Lax-Friedrichs flux with zero numerical viscosity is considered, i.e., $p=\frac{1}{2} p+\frac{1}{2} p$, otherwise the numerical diffusion will become large when $\varepsilon \rightarrow 0$ [10].

Remark 5 For the explicit time discretization, the CFL stability condition is described as

$$
\Delta t \leq C F L \cdot h / \max _{\mathbf{u} \cdot \mathbf{n}, \rho}\left(|\mathbf{u} \cdot \mathbf{n}|+\sqrt{p^{\prime}(\rho)} / \varepsilon\right)=O(\varepsilon) \stackrel{\varepsilon \rightarrow 0}{\longrightarrow} 0
$$

which leads to unaffordable computational costs in the low Mach number regime. For the IMEX-ARSp time discretization, the convective CFL stability condition is obtained as

$$
\Delta t \leq C F L \cdot h / \max _{\mathbf{u} \cdot \mathbf{n}}\{2|\mathbf{u} \cdot \mathbf{n}|\}
$$

due to semi-implicit nature of the acoustic/advection splitting strategy. In order to avoid an excessively large time-step sizes when the velocity of the fluid is too small, Boscarino et al. [3] recommended the following stability restriction

$$
\Delta t \leq C F L \cdot h / \max _{\mathbf{u} \cdot \mathbf{n}, \rho}\left(|\mathbf{u} \cdot \mathbf{n}|+\min (1,1 / \varepsilon) \sqrt{p^{\prime}(\rho)}\right) .
$$

The stability conditions (38) and (39) of the IMEX-ARSp method are independent of the Mach number $\varepsilon$ and thus are less restrictive contrary to a standard explicit method. Therefore, the IMEX-ARSp method is more efficient than the explicit method for low-Mach number flows.

Remark 6 For one-dimensional case, the term $\Delta p=p_{x x}$ in (33) is approximated by a fourth-order central-type difference scheme:

$$
p_{i}^{\prime \prime}=\frac{-p_{i-2}+16 p_{i-1}-30 p_{i}+16 p_{i+1}-p_{i+2}}{12 h^{2}} .
$$

For two-dimensional case, this central-type difference scheme in a dimension-bydimension form is applied to $\triangle p=p_{x x}+p_{y y}$.

\section{AP property}

Definition 3 A scheme for the low Mach number limit of the Euler equations is asymptotic preserving, if it serves as a consistent and stable discretisation, independent of the Mach number $\varepsilon$.

Proposition 1 Consider the system (1) subject to well-prepared initial data (i.e., $\rho_{0}^{0}=$ const, $\nabla \cdot \mathbf{u}_{0}^{0}(\mathbf{x})=0$ ) and periodic, wall or homogeneous Neumann boundary conditions. The spatial derivatives are assumed to be continuous. The GSA IMEX-ARSp scheme ((29)(32)) (see Definitions 1 and 2) is used for the time discretization of the system (1) from $t=t^{0}$ to $t^{1}$ with the time-step size $\Delta t=t^{1}-t^{0}$ given by the stability conditions (38) or (39) independent of $\varepsilon$, and we then have

$$
\rho^{1}\left(\mathbf{x}, t^{1}\right) \stackrel{\varepsilon \rightarrow 0}{\longrightarrow} \rho_{0}^{0}, \nabla \cdot \mathbf{u}^{1}\left(\mathbf{x}, t^{1}\right) \stackrel{\varepsilon \rightarrow 0}{\longrightarrow} 0,
$$

and

$$
\mathbf{u}^{1}\left(\mathbf{x}, t^{1}\right) \stackrel{\varepsilon \rightarrow 0}{\longrightarrow} \mathbf{u}_{e}\left(\mathbf{x}, t^{1}\right)+O\left(\Delta t^{p+1}\right)
$$

Here, $\mathbf{u}_{e}$ is the exact solution of Eq. (5) with initial data $\mathbf{u}_{e}(\mathbf{x}, 0)=\mathbf{u}_{0}^{0}(\mathbf{x})$. 
Let $\mathbf{f}=\nabla \cdot \frac{\mathbf{q} \otimes \mathbf{q}}{\rho}, \vec{r}=\left(r^{(1)}, \ldots, r^{(s)}\right)^{T}$ for $r=\rho$ or $p$, and $\overrightarrow{\mathbf{v}}=\left(\mathbf{v}^{(1)}, \ldots, \mathbf{v}^{(s)}\right)^{T}$ for $\mathbf{v}=\mathbf{f}$ or $\mathbf{q}$ generated at the IMEX-ARSp intermediate stages $t_{k}=t^{0}+c_{k} \Delta t$. Following [10], we give the proof process of Proposition 1.

Proof Based on (29)-(32), we obtain from $t=t^{0}$ to $t^{1}$

$$
\begin{aligned}
& \vec{\rho}=\rho^{0} \mathbf{e}-\Delta t \mathbf{A} \nabla \cdot \overrightarrow{\mathbf{q}}, \\
& \overrightarrow{\mathbf{q}}=\mathbf{q}^{0} \mathbf{e}-\Delta t \tilde{\mathbf{A}} \nabla \cdot \overrightarrow{\mathbf{f}}-\frac{\Delta t}{\varepsilon^{2}} \mathbf{A} \nabla \vec{p},
\end{aligned}
$$

and

$$
\begin{aligned}
\rho^{1} & =\rho^{0}-\Delta t \mathbf{b}^{T} \nabla \cdot \overrightarrow{\mathbf{q}} \\
\mathbf{q}^{1} & =\mathbf{q}^{0}-\Delta t \tilde{\mathbf{b}}^{T} \nabla \cdot \overrightarrow{\mathbf{f}}-\frac{\Delta t}{\varepsilon^{2}} \mathbf{b}^{T} \nabla \vec{p},
\end{aligned}
$$

where $\mathbf{e}=(1, \ldots, 1)^{T} \in \mathbb{R}^{s}$.

Taking the divergence of both sides of (44) and substituting $\nabla \cdot \overrightarrow{\mathbf{q}}$ into (43) yields a pressure Poisson equation

$$
\frac{\Delta t^{2}}{\varepsilon^{2}} \mathbf{A}^{2} \Delta \vec{p}=\left(\vec{\rho}-\rho^{0} \mathbf{e}\right)+\Delta t \mathbf{A} \nabla \cdot \mathbf{q}^{0} \mathbf{e}-\Delta t^{2} \mathbf{A} \tilde{\mathbf{A}} \nabla \cdot(\nabla \cdot \overrightarrow{\mathbf{f}}) .
$$

In order to study the asymptotic behavior of the solutions, we plug the $\varepsilon$-asymptotic expansions $f(\mathbf{x})=f_{0}(\mathbf{x})+\varepsilon^{2} f_{2}(\mathbf{x})+O\left(\varepsilon^{3}\right)$ for variables $\vec{\rho}, \rho^{1}, \vec{p}, p^{1}$ and $g(\mathbf{x})=g_{0}(\mathbf{x})+O\left(\varepsilon^{2}\right)$ for variables $\overrightarrow{\mathbf{q}}, \mathbf{q}^{1}$ into (43)-(47). Note that the term $O(\varepsilon)$ does not appear in Eq. (1). At the leading order $O\left(\varepsilon^{-2}\right)$ terms in (44), we obtain $\nabla \vec{p}_{0}=\nabla \vec{p}\left(\vec{\rho}_{0}\right)=0$. Therefore, $\vec{p}_{0}$ and $\vec{\rho}_{0}$ are independent of $\mathbf{x}$. Collecting the order $O(1)$ terms in $\varepsilon$ generates

$$
\begin{aligned}
\vec{\rho}_{0}-\rho_{0}^{0} \mathbf{e} & =-\Delta t \mathbf{A} \nabla \cdot \overrightarrow{\mathbf{q}}_{0}, \\
\overrightarrow{\mathbf{q}}_{0}-\mathbf{q}_{0}^{0} \mathbf{e} & =-\Delta t \tilde{\mathbf{A}} \nabla \cdot \overrightarrow{\mathbf{f}}_{0}-\Delta t \mathbf{A} \nabla \vec{p}_{2}, \\
\rho_{0}^{1}-\rho_{0}^{0} & =-\Delta t \mathbf{b}^{T} \nabla \cdot \overrightarrow{\mathbf{q}}_{0}, \\
\mathbf{q}_{0}^{1}-\mathbf{q}_{0}^{0} & =-\Delta t \tilde{\mathbf{b}}^{T} \nabla \cdot \overrightarrow{\mathbf{f}}_{0}-\Delta t \mathbf{b}^{T} \nabla \vec{p}_{2}, \\
\Delta t^{2} \mathbf{A}^{2} \Delta \vec{p}_{2} & =\vec{\rho}_{0}-\rho_{0}^{0} \mathbf{e}+\Delta t \mathbf{A} \nabla \cdot \mathbf{q}_{0}^{0} \mathbf{e}-\Delta t^{2} \mathbf{A} \tilde{\mathbf{A}} \nabla \cdot\left(\nabla \cdot \overrightarrow{\mathbf{f}}_{0}\right),
\end{aligned}
$$

where $\mathbf{q}_{0}^{0}=\rho_{0}^{0} \mathbf{u}_{0}^{0}$ and $\overrightarrow{\mathbf{f}}_{0}=\overrightarrow{\mathbf{q}}_{0} \otimes \overrightarrow{\mathbf{q}}_{0} / \vec{\rho}_{0}$.

Integrating (48) over the whole computational domain and applying periodic, wall or homogeneous Neumann boundary conditions yields $\vec{\rho}_{0}=\rho_{0}^{0} \mathbf{e}$, i.e., $\vec{\rho}_{0}$ is independent of $t$. Since $\vec{\rho}_{0}=\rho_{0}^{0} \mathbf{e}$ and $\nabla \cdot \mathbf{q}_{0}^{0}=\rho_{0}^{0} \nabla \cdot \mathbf{u}_{0}^{0}=0$, (52) becomes

$$
\mathbf{A}^{2} \triangle \vec{p}_{2}=-\mathbf{A} \tilde{\mathbf{A}} \nabla \cdot\left(\nabla \cdot \overrightarrow{\mathbf{f}}_{0}\right) .
$$

Based on Definition 1, (53) can be simplified as

$$
\triangle \vec{p}_{2}^{\prime}=-A_{22}^{-1}\left(\tilde{A}_{21} \nabla \cdot\left(\nabla \cdot \overrightarrow{\mathbf{f}}_{0}^{(1)}\right)+\tilde{A}_{22} \nabla \cdot\left(\nabla \cdot \overrightarrow{\mathbf{f}}_{0}^{\prime}\right)\right),
$$

where $\vec{p}^{\prime}=\left(p^{(2)}, \ldots, p^{(s)}\right)^{T}$ and $\overrightarrow{\mathbf{f}}_{0}^{\prime}=\left(\mathbf{f}^{(2)}, \ldots, \mathbf{f}^{(s)}\right)^{T}$.

Taking the divergence of both sides of (49) and applying Definition 1 and (54) yields

$$
\nabla \cdot \overrightarrow{\mathbf{q}}_{0}=0, \nabla \cdot \overrightarrow{\mathbf{u}}_{0}=0
$$

Based on (50) and (55), we have $\rho_{0}^{1}=\rho_{0}^{0}$, i.e., $\rho^{1}\left(\mathbf{x}, t^{1}\right) \stackrel{\varepsilon \rightarrow 0}{\longrightarrow} \rho_{0}^{0}$. Taking the divergence of both sides of (51) and applying Definition 2 generates

$$
\nabla \cdot \mathbf{q}_{0}^{1}=-\Delta t \mathbf{e}_{s}^{T}\left(\tilde{\mathbf{A}} \nabla \cdot\left(\nabla \cdot \overrightarrow{\mathbf{f}}_{0}\right)+\mathbf{A} \triangle \vec{p}_{2}\right) .
$$


By applying Definition 1 and (54), (56) is simplified as $\nabla \cdot \mathbf{q}_{0}^{1}=\rho^{1} \nabla \cdot \mathbf{u}_{0}^{1}=0$. Since $\rho_{0}^{1}=\rho_{0}^{0}$, we have $\nabla \cdot \mathbf{u}_{0}^{1}=0$, i.e., $\nabla \cdot \mathbf{u}^{1}\left(\mathbf{x}, t^{1}\right) \stackrel{\varepsilon \rightarrow 0}{\longrightarrow} 0$.

Both sides of (49) and (51) are divided by the constant density $\rho_{0}^{0}$ and we obtain the asymptotic limit of scheme for the incompressible Euler system (5):

$$
\begin{aligned}
& \overrightarrow{\mathbf{u}}_{0}=\mathbf{u}_{0}^{0} \mathbf{e}-\Delta t \tilde{\mathbf{A}} \frac{1}{\rho_{0}^{0}} \nabla \cdot \overrightarrow{\mathbf{f}}_{0}-\Delta t \mathbf{A} \frac{1}{\rho_{0}^{0}} \nabla \vec{p}_{2}, \\
& \mathbf{u}_{0}^{1}=\mathbf{u}_{0}^{0}-\Delta t \tilde{\mathbf{b}}^{T} \frac{1}{\rho_{0}^{0}} \nabla \cdot \overrightarrow{\mathbf{f}}_{0}-\Delta t \mathbf{b}^{T} \frac{1}{\rho_{0}^{0}} \nabla \vec{p}_{2},
\end{aligned}
$$

where $\vec{p}_{2}$ is given by (53). If the IMEX-ARSp scheme that has $p^{t h}$-order temporal accuracy is adopted to solve the system (5) subject to initial data $\mathbf{u}_{e}(\mathbf{x}, 0)=\mathbf{u}_{0}^{0}$, the asymptotic accuracy can be attained, i.e., (42) is proved.

Remark 7 A similar procedure can be adopted when going from $t^{n}$ to $t^{n+1}$ to prove that the limiting IMEX-ARSp scheme is a consistent discretization of the limiting equation for fixed $h$ and $\Delta t$. The IMEX-ARSp scheme is also asymptotically stable with the time-step restrictions (38) or (39) independent of the Mach number $\varepsilon$.

Remark 8 The AP property of the GSA IMEX-ARSp scheme is proved based on the assumption that the Euler equations are subject to well-prepared initial data. For the $S A$ IMEX-ARSp scheme, the AP property can also be proved using a similar procedure under the same assumption. The proof of the AP property of the GSA IMEX-ARSp scheme combined with the fifth-order WCNS is relatively complex and has not been performed. In the next section, we will verify numerically the AP property of the IMEX-ARSp WCNS. In addition, the IMEX-ARSp scheme combined some other shock capturing schemes, e.g., the MUSCL (monotone upstream-centered scheme for conservation laws) type finite volume scheme proposed in [44], can also maintain numerically the AP property.

\section{Numerical examples}

The third-order GSA IMEX-ARS3 WCNS is applied to solve the following numerical examples. In some convergence tests, the third-order SA IMEX-ARS3 WCNS is also tested. See Appendix for the characteristics of the GSA and SA IMEX-ARS3 scheme. In addition, the third-order explicit total variation diminishing Runge-Kutta time-stepping method [30] coupled with the fifth-order WCNS (TVD-RK3 WCNS) and the thirdorder GAS IMEX-ARS3 scheme coupled with the third-order MUSCL type finite volume scheme (IMEX-ARS3 MUSCL) are used to solve some examples for comparisons.

The order of temporal accuracy is numerically calculated as

$$
p=\log _{2}\left(E_{\Delta t_{1}} / E_{\Delta t_{2}}\right),
$$

where $E_{\Delta t_{1}}$ obtained with $\Delta t_{1}=O(h)$ and $E_{\Delta t_{2}}$ obtained with $\Delta t_{2}=\Delta t_{1} / 2$ are the global errors in the $L^{\infty}$ and $L^{1}$ norms, respectively. The space-step size $h$ deceases with the timestep size $\Delta t$. In the convergence tests, to test the temporal order of accuracy the time-step size is taken as $\Delta t=C F L \cdot h(C F L=0.1)$, while it is set as $\Delta t=C F L \cdot h^{2}(C F L=0.1)$ to test the spatial order of accuracy. In the other tests, the time-step sizes of the IMEXARS3 WCNS and the IMEX-ARS3 MUSCL are computed by the stability conditions (38) or (39), while the time-step size of the TVD-RK3 WCNS is calculated by the stability condition (37). 
Table 1 Example 1. $L^{\infty}$ errors and numerical orders of temporal accuracy obtained with the GSA IMEX-ARS3 WCNS at $t=0.1$

\begin{tabular}{|c|c|c|c|c|c|c|}
\hline \multirow{2}{*}{ N } & \multicolumn{2}{|l|}{$\varepsilon=0.01$} & \multicolumn{2}{|l|}{$\varepsilon=0.1$} & \multicolumn{2}{|l|}{$\varepsilon=1$} \\
\hline & $L^{\infty}$ error & Order & $L^{\infty}$ error & Order & $L^{\infty}$ error & Order \\
\hline 40 & $7.59 E-03$ & & $7.16 E-05$ & & $1.23 E-03$ & \\
\hline 80 & $3.03 E-03$ & 1.32 & $8.78 E-06$ & 3.03 & $9.95 E-05$ & 3.63 \\
\hline 160 & $6.37 E-04$ & 2.25 & $1.17 E-06$ & 2.90 & $1.31 E-05$ & 2.92 \\
\hline 320 & $9.85 E-05$ & 2.69 & $1.48 E-07$ & 2.99 & $6.58 E-07$ & 4.32 \\
\hline 640 & $1.28 E-05$ & 2.94 & $1.95 E-08$ & 2.93 & $8.75 E-09$ & 6.23 \\
\hline
\end{tabular}

Example 1 Convergence test for one-dimensional case [3]

This example is adopted to test the temporal order of accuracy of the GSA and SA IMEX-ARS3 WCNS for one-dimensional case. The computational domain is [-2.5, 2.5] with periodic boundary conditions. The initial data for one-dimensional isentropic Euler equations (1) is given by

$$
u(x, 0)=\sin \left(\frac{2 \pi x}{L}\right), \rho(x, 0)=\left(1+\frac{(\gamma-1) u(x, 0)}{c}\right)^{2}, p=\rho^{\gamma},
$$

where $\gamma=2, c=2 \sqrt{\gamma} / \varepsilon, L=5$ and the final time is fixed to $T_{f}=0.1$. To measure the temporal convergence of the GSA and SA IMEX-ARS3 WCNS, the solution computed on a fine mesh with $N=2560$ cells is used as a reference ("exact") solution since the exact solution of this problem is unknown. Tables 1 and 2 report the numerical $L^{\infty}$ and $L^{1}$-norm errors and orders of accuracy in time for the density obtained with the GSA IMEXARS3 WCNS at different Mach numbers, while Tables 3 and 4 show the corresponding results obtained with the SA IMEX-ARS3 WCNS. It is observed that both methods show a third-order temporal convergence, as expected, for different values of the Mach number, from compressible to incompressible regimes. In the following examples, we only investigate the performance of the GSA IMEX-ARS scheme. Here, we also test the spatial accuracy of the GSA IMEX-ARS3 WCNS. The numerical $L^{\infty}$ and $L^{1}$-norm errors are computed by comparing the numerical solutions of the density on third consecutive mesh sizes [13]. Tables 5 and 6 report the corresponding results at $t=0.05$, which demonstrate a fifth-order spatial convergence of the GSA IMEX-ARS3 WCNS at different Mach numbers.

Example 2 One-dimensional Riemann problem $[4,6,10]$

This example that consists in several interacting Riemann problems is used to validate the asymptotic stability of the IMEX-ARS3 WCNS. The computational domain is $[0,1]$ with periodic boundary conditions. The initial data is described as

Table 2 Example 1. $L^{1}$ errors and numerical orders of temporal accuracy obtained with the GSA IMEX-ARS3 WCNS at $t=0.1$

\begin{tabular}{|c|c|c|c|c|c|c|}
\hline \multirow{2}{*}{ N } & \multicolumn{2}{|l|}{$\varepsilon=0.01$} & \multicolumn{2}{|l|}{$\varepsilon=0.1$} & \multicolumn{2}{|l|}{$\varepsilon=1$} \\
\hline & $L^{1}$ error & Order & $L^{1}$ error & Order & $L^{1}$ error & Order \\
\hline 40 & $4.68 E-03$ & & $2.59 E-05$ & & $2.18 E-04$ & \\
\hline 80 & $1.63 E-03$ & 1.52 & $3.19 E-06$ & 3.02 & $1.18 E-05$ & 4.22 \\
\hline 160 & $2.79 E-04$ & 2.55 & $4.00 E-07$ & 2.99 & $5.48 E-07$ & 4.42 \\
\hline 320 & $3.88 E-05$ & 2.85 & $5.02 E-08$ & 3.00 & $1.80 E-08$ & 4.93 \\
\hline 640 & $4.94 E-06$ & 2.97 & $6.34 E-09$ & 2.98 & $1.40 E-09$ & 3.69 \\
\hline
\end{tabular}


Table 3 Example 1. $L^{\infty}$ errors and numerical orders of temporal accuracy obtained with the SA IMEX-ARS3 WCNS at $t=0.1$

\begin{tabular}{|c|c|c|c|c|c|c|}
\hline \multirow{2}{*}{$N$} & \multicolumn{2}{|l|}{$\varepsilon=0.01$} & \multicolumn{2}{|l|}{$\varepsilon=0.1$} & \multicolumn{2}{|l|}{$\varepsilon=1$} \\
\hline & $L^{\infty}$ error & Order & $L^{\infty}$ error & Order & $L^{\infty}$ error & Order \\
\hline 40 & $7.90 E-03$ & & $9.18 E-05$ & & $6.50 E-04$ & \\
\hline 80 & $3.34 E-03$ & 1.24 & $1.09 E-05$ & 3.07 & $9.50 E-05$ & 2.78 \\
\hline 160 & $7.45 E-04$ & 2.16 & $1.40 E-06$ & 2.97 & $1.71 E-05$ & 2.47 \\
\hline 320 & $1.19 E-04$ & 2.65 & $1.75 E-07$ & 3.00 & $1.97 E-07$ & 6.44 \\
\hline 640 & $1.57 E-05$ & 2.92 & $2.19 E-08$ & 3.00 & $6.74 E-10$ & 8.19 \\
\hline
\end{tabular}

$$
\left\{\begin{array}{l}
\rho(x, 0)=1, \quad q(x, t)=1-0.5 \varepsilon^{2}, \quad x \in[0,0.2] \cup[0.8,1] \\
\rho(x, 0)=1+\varepsilon^{2}, q(x, t)=1, \quad x \in[0.2,0.3] \\
\rho(x, 0)=1, \quad q(x, t)=1+0.5 \varepsilon^{2}, \quad x \in[0.3,0.7] \\
\rho(x, 0)=1-\varepsilon^{2}, q(x, t)=1, \quad x \in[0.7,0.8]
\end{array}\right.
$$

and the pressure is $p(\rho)=\rho^{2}$. The time-step size is computed by (38) with $C F L=0.3$ and the final time is fixed to $T_{f}=0.05$. The values of the Mach number are chosen as $\varepsilon=\sqrt{0.99}, 0.3$ and 0.01 corresponding to the compressible, intermediate and incompressible regimes, respectively. The numerical solutions calculated by the GSA IMEX-ARS3 WCNS and the GSA IMEX-ARS3 MUSCL with $N=200$ cells are compared to a reference solution computed by the explicit TVD-RK3 WCNS with $N=2000$ cells. Figure 1 illustrates the solutions for the density and momentum at different Mach numbers. From this figure, we observe the IMEX-ARS3 WCNS and the TVD-RK3 WCNS can capture shocks and contact discontinuities that are stronger when $\varepsilon$ is bigger, while the IMEX-ARS3 MUSCL suffers from excessive numerical diffusion. Spurious oscillations are observed with the TVD-RK3 WCNS when $\varepsilon=\sqrt{0.99}$ (see Fig. 1a and b). The utilization of WENO interpolation based on the characteristic variables instead of the flux functions can effectively suppress spurious oscillations near discontinuities. Compared to the TVD-RK3 WCNS, the IMEX-ARS3 WCNS and the IMEX-ARS3 MUSCL capture the limit incompressible solution faster when $\varepsilon$ is small. When $\varepsilon$ approaches 0 , the TVD-RK3 WCNS can not capture the correct solution, due to the excessive numerical viscosity.

Example 3 Sod shock tube problem [5]

This problem with non-well prepared initial data is used to investigate the performance of the GSA IMEX-ARS3 WCNS in the intermediate and incompressible regimes. The com-

Table 4 Example 1. $L^{1}$ errors and numerical orders of temporal accuracy obtained with the SA IMEX-ARS3 WCNS at $t=0.1$

\begin{tabular}{|c|c|c|c|c|c|c|}
\hline \multirow{2}{*}{ N } & \multicolumn{2}{|l|}{$\varepsilon=0.01$} & \multicolumn{2}{|l|}{$\varepsilon=0.1$} & \multicolumn{2}{|l|}{$\varepsilon=1$} \\
\hline & $L^{1}$ error & Order & $L^{1}$ error & Order & $L^{1}$ error & Order \\
\hline 40 & $4.87 E-03$ & & $3.10 E-05$ & & $1.05 E-04$ & \\
\hline 80 & $1.79 E-03$ & 1.45 & $3.94 E-06$ & 2.98 & $8.36 E-06$ & 3.65 \\
\hline 160 & $3.29 E-04$ & 2.44 & $4.86 E-07$ & 3.02 & $4.77 E-07$ & 4.13 \\
\hline 320 & $4.73 E-05$ & 2.80 & $6.08 E-08$ & 3.00 & $5.39 E-09$ & 6.47 \\
\hline 640 & $6.10 E-06$ & 2.95 & $7.53 E-09$ & 3.01 & $1.27 E-10$ & 5.41 \\
\hline
\end{tabular}


Table 5 Example 1. $L^{\infty}$ errors and numerical orders of spatial accuracy obtained with the GSA IMEX-ARS3 WCNS at $t=0.05$

\begin{tabular}{|c|c|c|c|c|c|c|}
\hline \multirow{2}{*}{ N } & \multicolumn{2}{|l|}{$\varepsilon=0.01$} & \multicolumn{2}{|l|}{$\varepsilon=0.1$} & \multicolumn{2}{|l|}{$\varepsilon=1$} \\
\hline & $L^{\infty}$ error & Order & $L^{\infty}$ error & Order & $L^{\infty}$ error & Order \\
\hline 20 & $8.70 E-03$ & & $3.22 E-04$ & & $4.49 E-03$ & \\
\hline 40 & $1.80 E-04$ & 5.59 & $1.30 E-05$ & 4.63 & $5.49 E-04$ & 3.03 \\
\hline 80 & $2.83 E-06$ & 5.99 & $2.61 E-07$ & 5.64 & $6.68 E-05$ & 3.04 \\
\hline 160 & $4.36 E-08$ & 6.02 & $9.34 E-10$ & 8.13 & $1.96 E-06$ & 5.09 \\
\hline 320 & $6.59 E-10$ & 6.05 & $1.08 E-10$ & 3.11 & $2.44 E-09$ & 9.65 \\
\hline
\end{tabular}

putational domain is $[0,1]$ with zero Neumann boundary conditions. The pressure is $p(\rho)=\rho$. The non-well prepared initial data including discontinuity is

$$
(\rho, u)= \begin{cases}(1.000,0.000), & x \leq 0.5, \\ (0.125,0.000), & \text { otherwise. }\end{cases}
$$

The time-step size is computed by (39) with CFL $=0.1$. The values of the Mach number are chosen as $\varepsilon=0.3$ and 0.03 corresponding to the intermediate and incompressible regimes, respectively. For both $\varepsilon=0.3$ and $\varepsilon=0.03$, reference solutions are generated using the GSA IMEX-ARS3 MUSCL with $N=2000$ cells. Figure 2 depicts the density and momentum calculated by the GSA IMEX-ARS3 WCNS with $N=200$ cells at time $T_{f}=0.05$ for $\varepsilon=0.3$ and at time $T_{f}=0.005$ for $\varepsilon=0.03$. From this figure, the density and momentum are not close to the solutions of the incompressible Euler equations. The solutions include the rarefaction waves moving to the left and shock waves moving to the right for both $\varepsilon=0.3$ and $\varepsilon=0.03$. From Fig. $2 a$ and $b$, it is observed that when $\varepsilon=0.3$, the numerical solution obtained with the IMEX-ARS3 WCNS has a good agreement with the reference solution. When $\varepsilon=0.03$ in the incompressible regime, nonphysical oscillations arise near discontinuities based on the IMEX-ARS3 WCNS (see Fig. $2 c$ and d). Nonphysical oscillations are a common phenomenon when shocks develop in the low Mach number regime $[4,5]$ and can be eliminated by decreasing $\Delta t$ and taking a smaller $C F L=0.01$.

Example 4 Convergence test for two-dimensional case [19]

This example is used to test the temporal order of accuracy of the GSA IMEXARS3 WCNS for two-dimensional case. The computational domain is $[0,1] \times[0,1]$ with periodic boundary conditions. The pressure is $p(\rho)=\frac{1}{2} \rho^{2}$. The final time is $T_{f}=0.02$. The initial data is given by the following exact solution

Table 6 Example 1. $L^{1}$ errors and numerical orders of spatial accuracy obtained with the GSA IMEX-ARS3 WCNS at $t=0.05$

\begin{tabular}{|c|c|c|c|c|c|c|}
\hline \multirow{2}{*}{ N } & \multicolumn{2}{|l|}{$\varepsilon=0.01$} & \multicolumn{2}{|l|}{$\varepsilon=0.1$} & \multicolumn{2}{|l|}{$\varepsilon=1$} \\
\hline & $L^{1}$ error & Order & $L^{1}$ error & Order & $L^{1}$ error & Order \\
\hline 20 & $5.58 E-04$ & & $4.21 E-05$ & & $1.10 E-03$ & \\
\hline 40 & $3.13 E-05$ & 4.16 & $6.63 E-07$ & 5.99 & $1.29 E-05$ & 6.42 \\
\hline 80 & $4.57 E-06$ & 2.78 & $5.99 E-09$ & 6.79 & $1.13 E-07$ & 6.83 \\
\hline 160 & $9.96 E-08$ & 5.52 & $9.91 E-12$ & 9.24 & $8.92 E-10$ & 6.98 \\
\hline 320 & $5.53 E-11$ & 10.81 & $9.86 E-13$ & 3.33 & $5.42 E-12$ & 7.36 \\
\hline
\end{tabular}




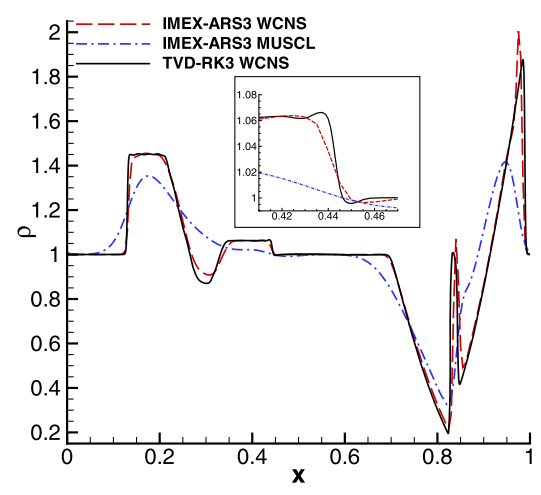

(a) Density, $\varepsilon=\sqrt{0.99}$

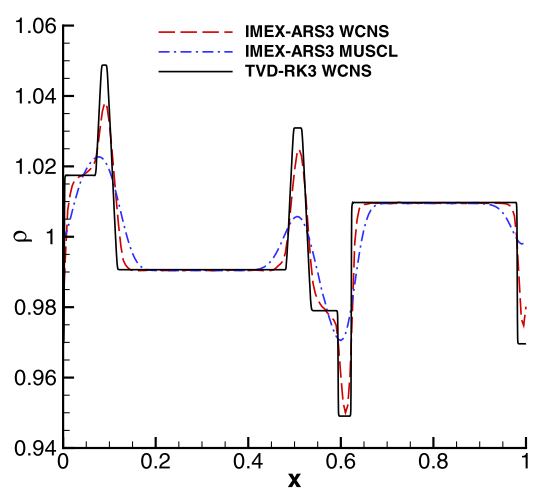

(c) Density, $\varepsilon=0.3$

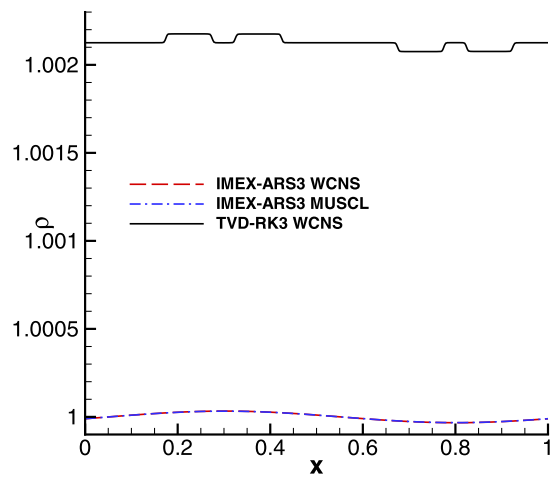

(e) Density, $\varepsilon=0.01$

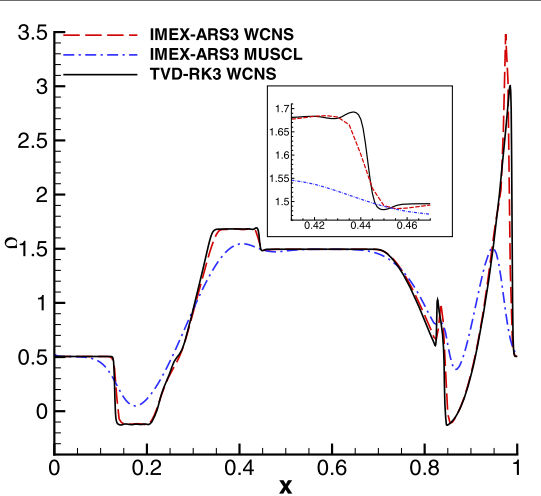

(b) Momentum, $\varepsilon=\sqrt{0.99}$

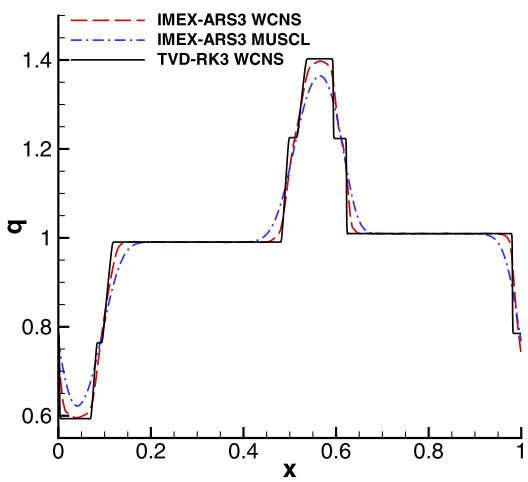

(d) Momentum, $\varepsilon=0.3$

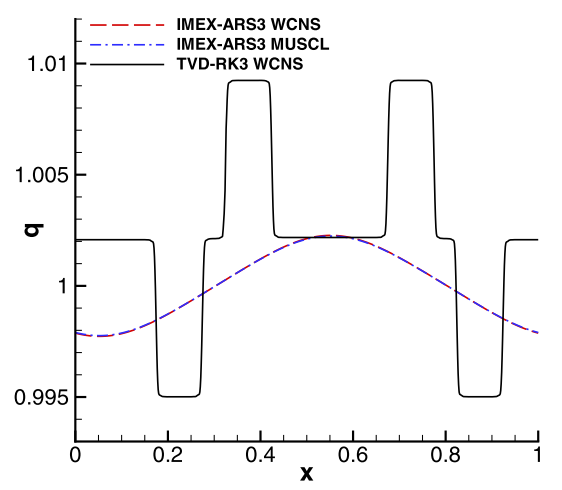

(f) Momentum, $\varepsilon=0.01$

Fig. 1 Example 2. Density (left) and momentum (right) calculated by the GSA IMEX-ARS3 WCNS and the GSA IMEX-ARS3 MUSCL with $N=200$ cells and the TVD-RK3 WCNS with $N=2000$ cells at $t=0.05$. The values of the Mach number are $\varepsilon=\sqrt{0.99}$ (top), 0.3 (middle) and 0.05 (bottom). a Density, $\varepsilon=\sqrt{0.99}$, b

Momentum, $\varepsilon=\sqrt{0.99}, \mathbf{c}$ Density, $\varepsilon=0.3$, d Momentum, $\varepsilon=0.3$, e Density, $\varepsilon=0.01$, f Momentum, $\varepsilon=0.01$

$$
\left\{\begin{array}{l}
\rho(\mathbf{x}, t)=1+\varepsilon^{2} \frac{1.5^{2}}{16 \pi^{2}} \delta(r)(k(r)-k(\pi)), \\
u(\mathbf{x}, t)=0.6+1.5(1+\cos (r)) \delta(r)(0.5-y), \\
v(\mathbf{x}, t)=1.5(1+\cos (r)) \delta(r)(x-0.6 t-0.5),
\end{array}\right.
$$




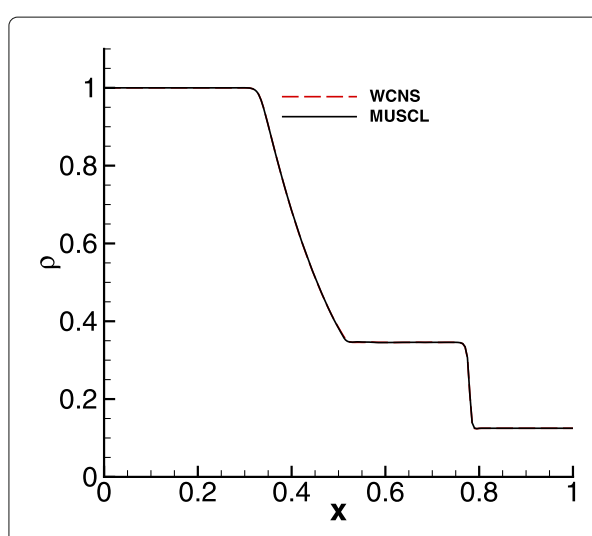

(a) Density, $\varepsilon=0.3$

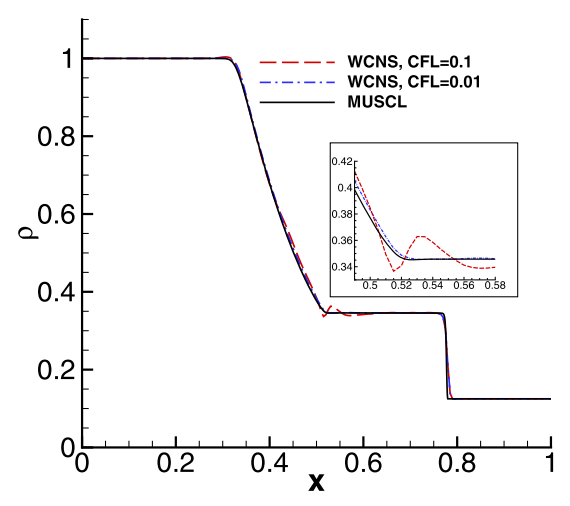

(c) Density, $\varepsilon=0.03$

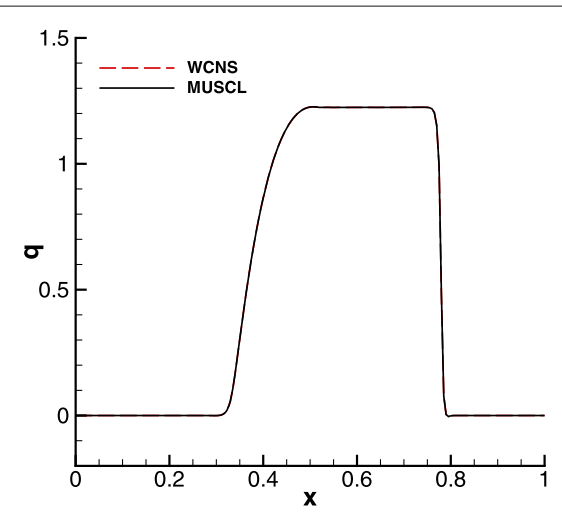

(b) Momentum, $\varepsilon=0.3$

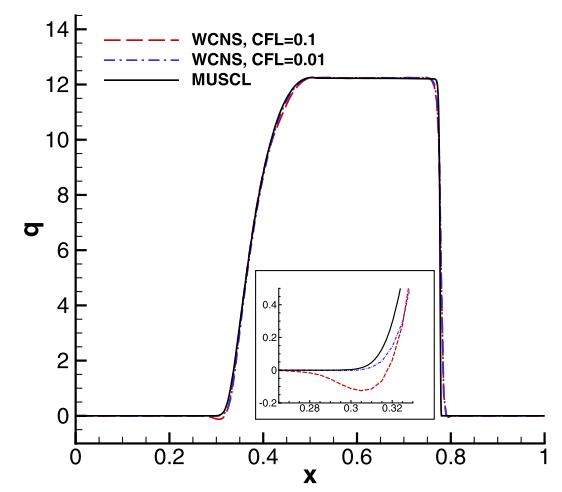

(d) Momentum, $\varepsilon=0.03$

Fig. 2 Example 3. Density (left) and momentum (right) calculated by the GSA IMEX-ARS3 WCNS with $N=200$ cells and the GSA IMEX-ARS3 MUSCL with $N=2000$ cells at $t=0.05$ for $\varepsilon=0.3$ and at $t=0.005$ for $\varepsilon=0.03$. The values of the Mach number are $\varepsilon=0.3$ (top) and 0.03 (bottom). $\mathbf{a}$ Density, $\varepsilon=0.3$. $\mathbf{b}$ Momentum, $\varepsilon=0.3$. c Density, $\varepsilon=0.03$. d Momentum, $\varepsilon=0.03$

where $r=4 \pi \sqrt{(x-0.6 t-0.5)^{2}+(y-0.5)^{2}}, k(r)=2 \cos (r)+2 r \sin (r)+0.125 \cos (2 r)+$ $0.25 r \sin (2 r)+0.75 r^{2}$ and

$$
\delta(r)= \begin{cases}1, & r<\pi \\ 0, & \text { otherwise }\end{cases}
$$

Tables 7 and 8 show the numerical $L^{\infty}$ and $L^{1}$-norm errors and orders of accuracy in time for the momentum ou based on different values of the Mach number, from compressible to incompressible regimes, which validates the GSA IMEX-ARS3 WCNS for two-dimensional case can attain the designed temporal accuracy. In addition, the $L^{1}$-norm convergence is better than the $L^{\infty}$-norm convergence under the same conditions.

Example 5 Two-dimensional Riemann problem $[8,10]$

This example is used to test the performance of the GSA IMEX-ARS3 WCNS at a high Mach number for two-dimensional case. The computational domain is $[0,1] \times[0,1]$ with 
Table 7 Example 4. $L^{\infty}$ errors and numerical orders of temporal accuracy obtained with the GSA IMEX-ARS3 WCNS at $t=0.02$

\begin{tabular}{|c|c|c|c|c|c|c|}
\hline \multirow{2}{*}{$M \times N$} & \multicolumn{2}{|l|}{$\varepsilon=0.01$} & \multicolumn{2}{|l|}{$\varepsilon=0.1$} & \multicolumn{2}{|l|}{$\varepsilon=1$} \\
\hline & $L^{\infty}$ error & Order & $L^{\infty}$ error & Order & $L^{\infty}$ error & Order \\
\hline $40 \times 40$ & $2.08 E-03$ & & $2.10 E-03$ & & $2.24 E-03$ & \\
\hline $80 \times 80$ & $8.02 E-04$ & 1.37 & $7.98 E-04$ & 1.40 & $7.76 E-04$ & 1.53 \\
\hline $160 \times 160$ & $3.01 E-04$ & 1.42 & $3.02 E-04$ & 1.40 & $3.02 E-04$ & 1.36 \\
\hline $320 \times 320$ & $5.64 E-05$ & 2.41 & $5.76 E-05$ & 2.39 & $6.53 E-05$ & 2.21 \\
\hline $640 \times 640$ & $8.36 E-06$ & 2.76 & $8.35 E-06$ & 2.79 & $8.36 E-06$ & 2.96 \\
\hline
\end{tabular}

zero Neumann boundary conditions. The pressure is $p(\rho)=\rho^{1.4}$. This initial data includes four shock waves and is described as

$$
(\rho, u, v)= \begin{cases}(0.1308,1.206,1.206), & x<0.5, y<0.5 \\ (0.5323,0.000,1.206), & x \geq 0.5, y<0.5 \\ (0.5323,1.206,0.000), & x<0.5, y \geq 0.5 \\ (1.5000,0.000,0.000), & \text { otherwise }\end{cases}
$$

The time-step size is computed by (38) with CFL $=0.4$. The values of the Mach number are chosen as $\varepsilon=1$ and 2 to test the robust shock-capturing capability of the IMEX-ARS3 WCNS in the compressible regime. Figure 3 plots the surfaces of the density profile obtained with the IMEX-ARS3 WCNS and the IMEX-ARS3 MUSCL on a mesh $50 \times 50$ at $T_{f}=0.1$. From this figure, we see that the IMEX-ARS3 WCNS has a higher resolution, compared to the IMEX-ARS3 MUSCL.

\section{Example 6 Cylindrical explosion problem $[6,11]$}

The computational domain is $[-1,1] \times[-1,1]$ with periodic boundary conditions and the pressure is given by $p(\rho)=\rho^{2}$. The initial density of the fluid is given by

$$
\left\{\begin{array}{l}
\rho(\mathbf{x}, 0)=1+\varepsilon^{2}, \quad \text { if } r<0.5, \\
\rho(\mathbf{x}, 0)=1, \quad \text { otherwise, }
\end{array}\right.
$$

where $r=\sqrt{x^{2}+y^{2}}$ is the distance to the center $(0,0)$ of the domain. The initial velocity of the fluid is given by

$$
\left\{\begin{array}{l}
u(\mathbf{x}, 0)=-\frac{x}{r} \frac{\beta}{\rho(\mathbf{x}, 0)}, \\
v(\mathbf{x}, 0)=-\frac{y}{r} \frac{\beta}{\rho(\mathbf{x}, 0)},
\end{array}\right.
$$

where $\beta=\max (0,1-r) e^{-16 r^{2}}$ and $(u, v)=(0,0)$, if $r \leq 10^{-15}$. The computational domain is divided into $50 \times 50$ cells. The values of the Mach number are chosen as $\varepsilon=1$

\begin{tabular}{|c|c|c|c|c|c|c|}
\hline \multirow{2}{*}{$M \times N$} & \multicolumn{2}{|l|}{$\varepsilon=0.01$} & \multicolumn{2}{|l|}{$\varepsilon=0.1$} & \multicolumn{2}{|l|}{$\varepsilon=1$} \\
\hline & $L^{1}$ error & Order & $L^{1}$ error & Order & $L^{1}$ error & Order \\
\hline $40 \times 40$ & $1.08 E-04$ & & $1.15 E-04$ & & $9.39 E-05$ & \\
\hline $80 \times 80$ & $2.31 E-05$ & 2.23 & $2.51 E-05$ & 2.20 & $2.13 E-05$ & 2.14 \\
\hline $160 \times 160$ & $4.04 E-06$ & 2.51 & $4.17 E-06$ & 2.59 & $4.10 E-06$ & 2.38 \\
\hline $320 \times 320$ & $3.87 E-07$ & 3.38 & $3.93 E-07$ & 3.41 & $4.04 E-07$ & 3.34 \\
\hline $640 \times 640$ & $4.10 E-08$ & 3.24 & $4.12 E-08$ & 3.25 & $6.37 E-08$ & 2.66 \\
\hline
\end{tabular}

Table 8 Example 4. $L^{1}$ errors and numerical orders of temporal accuracy obtained with the GSA IMEX-ARS3 WCNS at $t=0.02$ 


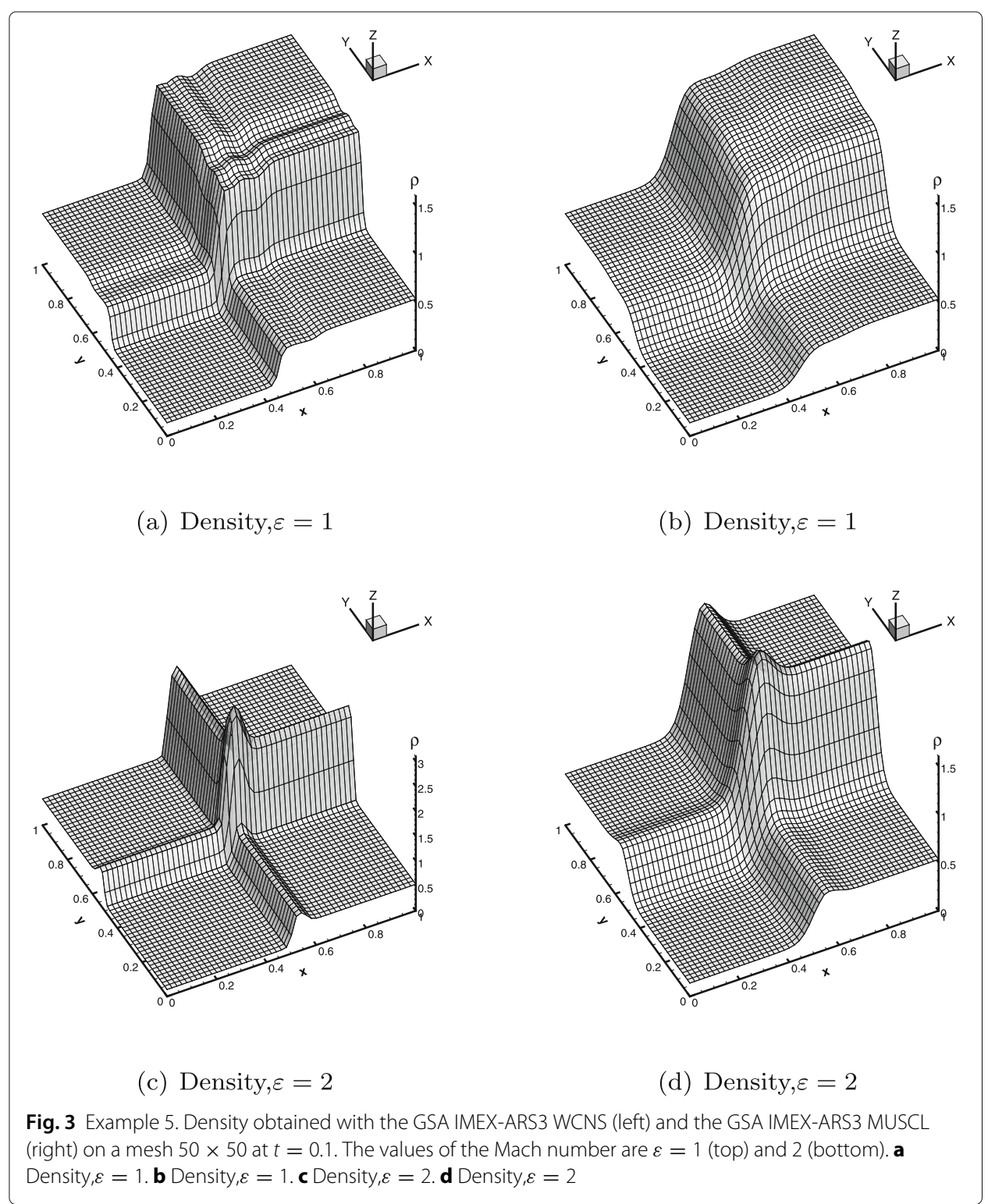

and 0.001. The time-step sizes are computed by (38) with CFL $=0.4$ for $\varepsilon=1$ and by (39) with $C F L=0.03$ for $\varepsilon=0.001$, respectively. Figure 4 plots the surfaces of the density profile and the velocity fields for $\varepsilon=1$ obtained with the GSA IMEX-ARS3 WCNS at different times. Figure 5 shows the density profile and the discrete divergence of the velocity $\nabla \cdot \mathbf{u}$ at time $t=0.05$ for a small value of $\varepsilon=0.001$. It is observed that when $\varepsilon=0.001$, the density converges to the constant value 1 and the divergence $\nabla \cdot \mathbf{u}$ is close to 0 .

Example 7 Shear flow problem $[3,10,16]$

This example is used to verify numerically the AP property of the IMEX-ARS3 WCNS in the low Mach number limit. The computational domain is $[0,2 \pi] \times[0,2 \pi]$ with periodic 


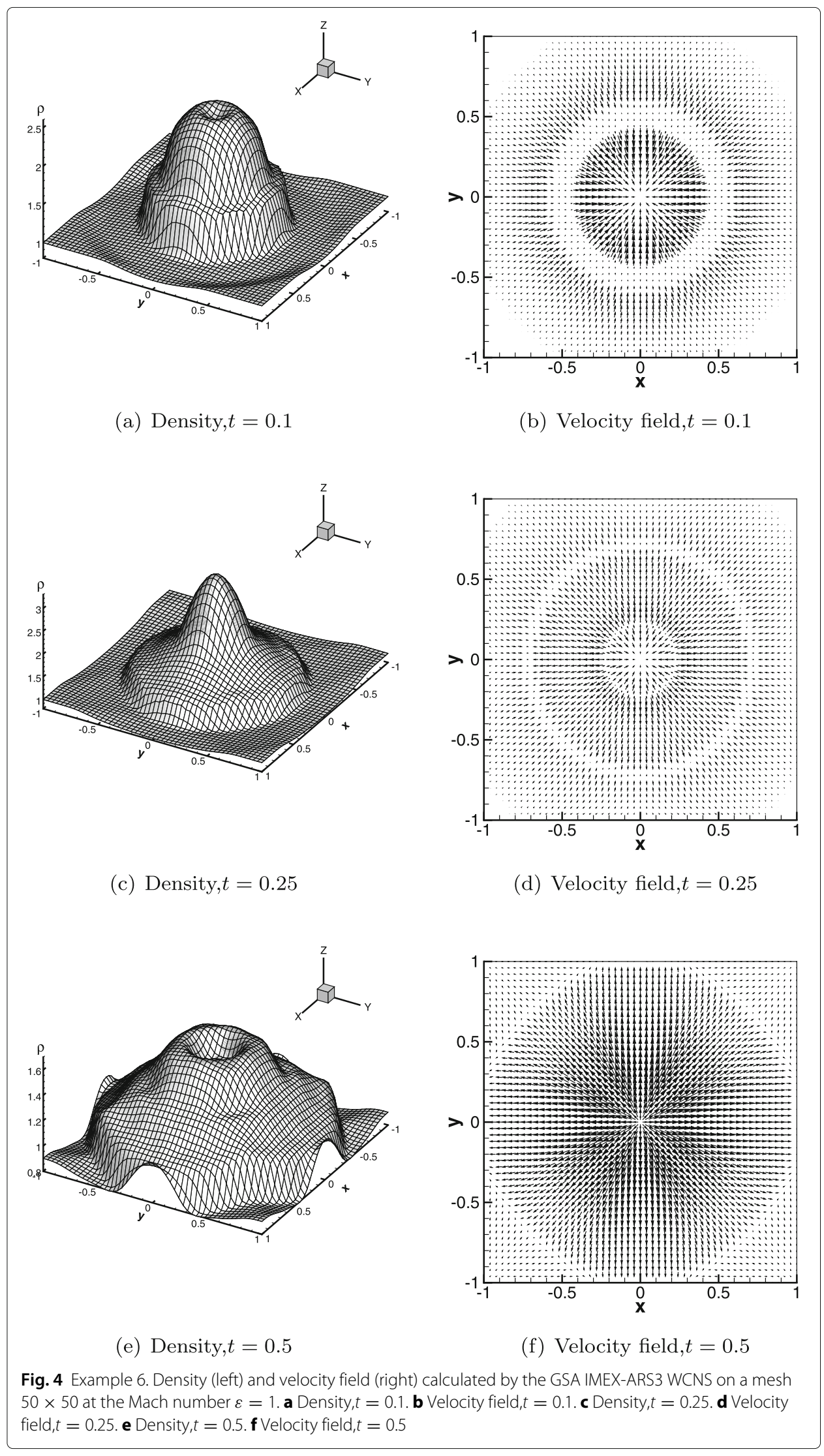




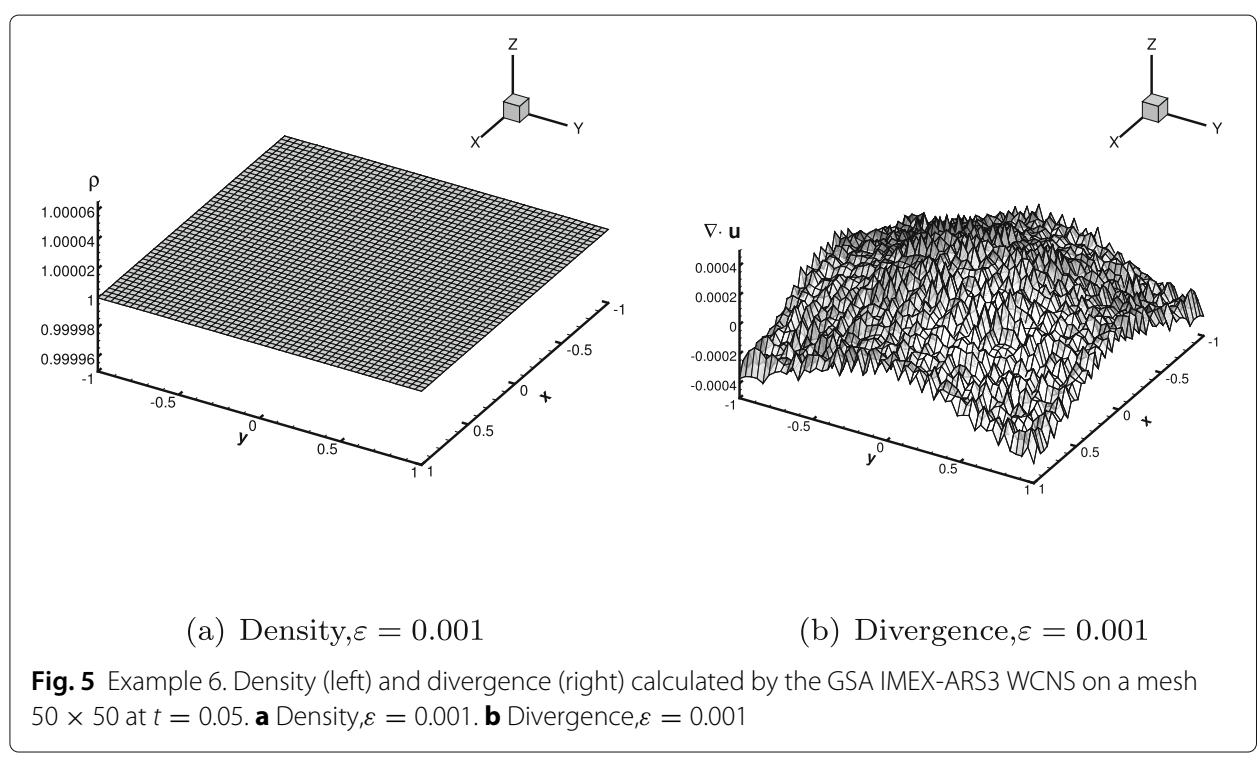

boundary conditions and the pressure is set to $p(\rho)=\rho^{1.4}$. Initially, a constant density and an incompressible velocity field are given by $\rho(\mathbf{x}, 0)=\pi / 15$ and

$$
u(\mathbf{x}, 0)=\left\{\begin{array}{l}
\tanh ((y-0.5 \pi) / \rho), \quad y \leq \pi, \\
\tanh ((1.5 \pi-y) / \rho), \text { otherwise, }
\end{array} \quad v(\mathbf{x}, 0)=0.05 \sin (x)\right.
$$

The time-step size is computed by (39) with $C F L=0.2$. The Mach number is $\varepsilon=10^{-4}$ and the final time is $T_{f}=10$. Figure 6 demonstrates the discrete vorticity $\omega=v_{x}-u_{y}$ on a mesh $50 \times 50$ at different times. From this figure, we see the vorticity quickly develops into roll-ups with smaller and smaller scales with the time evolution. Figure 7 shows the time history of the $L_{1}$-norm error of the discrete divergence of the velocity $\nabla \cdot \mathbf{u}$. It is observed that the $L_{1}$-norm error increases with time, which is in agreement with the phenomenon in [10] and is caused by the appearance of the smaller and smaller scale structures in the shear flow. The maximum error is smaller than that obtained with a finer mesh in [10].

\section{Example 8 Kelvin-Helmholtz instability problem [10]}

This problem is solved in the domain $[0,2 \pi] \times[0,4 \pi]$ with periodic boundary conditions. The pressure is $p(\rho)=\rho^{2}$ and the initial data reads

$$
\rho(\mathbf{x}, 0)=1, u(\mathbf{x}, 0)=\cos (y), \quad v(\mathbf{x}, 0)=0.03 \sin (0.5 x) .
$$

The time-step size is computed by (39) with $C F L=0.2$. The Mach number is $\varepsilon=10^{-3}$ and the final time is $T_{f}=40$. The discrete vorticity $\omega=v_{x}-u_{y}$ on a mesh $50 \times 50$ at different times is depicted in Fig. 8, which shows the emergence and subsequent evolution of small scale flow features. The time history of the $L_{1}$-norm error of the discrete divergence of the velocity $\nabla \cdot \mathbf{u}$ is shown in Fig. 9, from which we also observe that the $L_{1}$-norm error increases with time and the maximum error is smaller than that obtained with a finer mesh in [10]. 


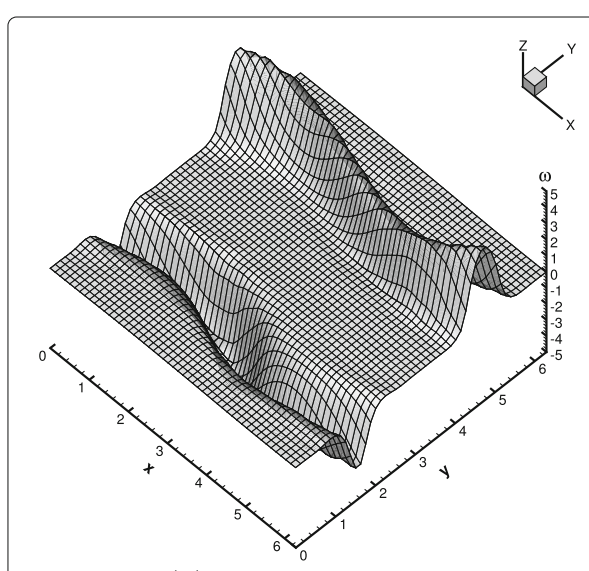

(a) Vorticity, $t=4$

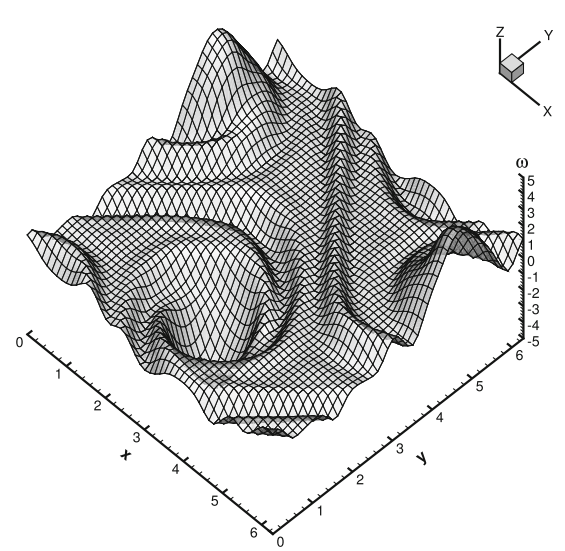

(c) Vorticity, $t=8$

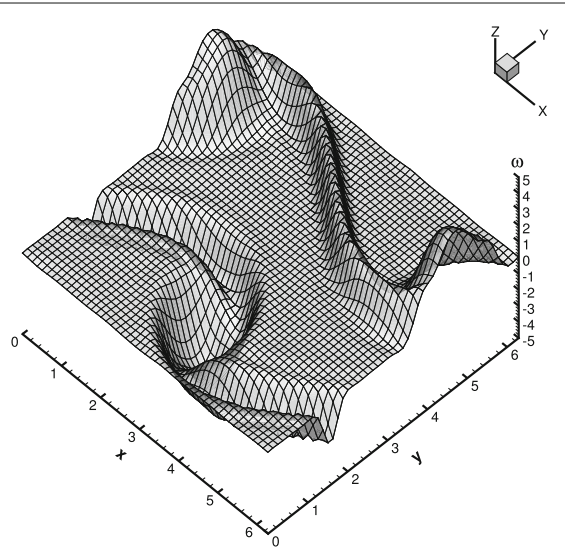

(b) Vorticity, $t=6$

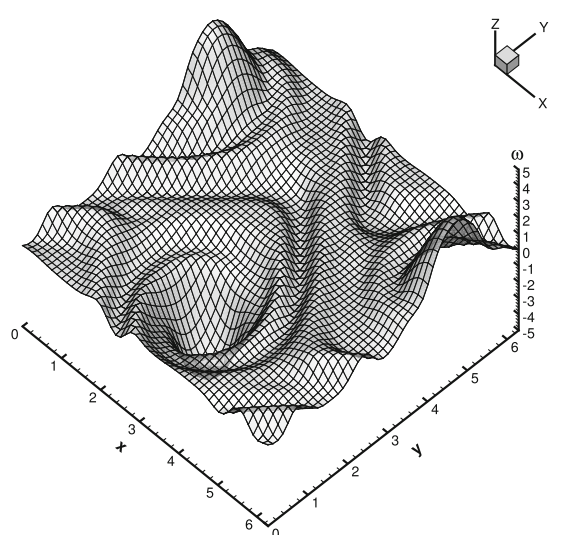

(d) Vorticity, $t=10$

Fig. 6 Example 7. Vorticity $\omega=v_{x}-u_{y}$ obtained with the GSA IMEX-ARS3 WCNS on a mesh $50 \times 50$ at different times. a Vorticity, $t=4$. $\mathbf{b}$ Vorticity, $t=6$. $\mathbf{c}$ Vorticity, $t=8$. d Vorticity, $t=10$

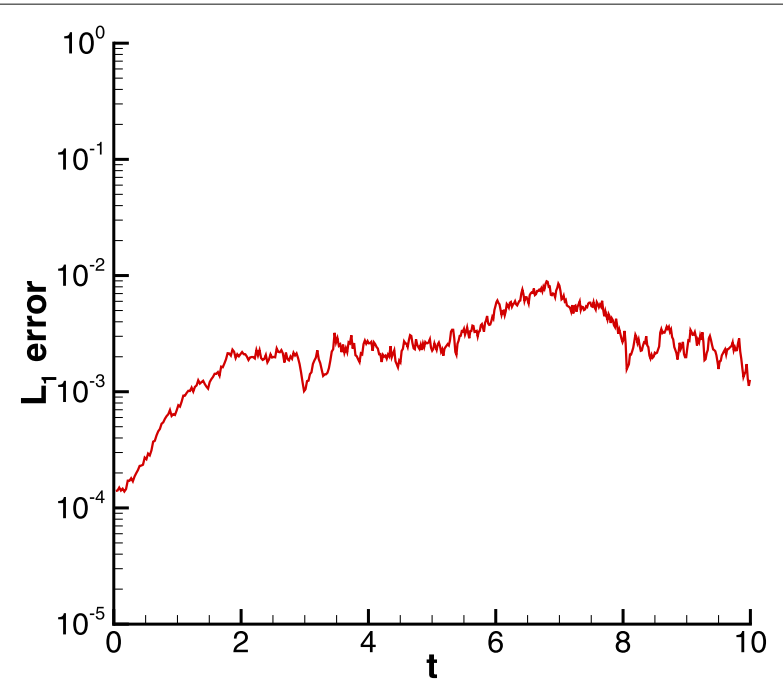

Fig. 7 Example 7. Time history of $L_{1}$ error of the divergence of the velocity $\nabla \cdot \mathbf{u}$ 


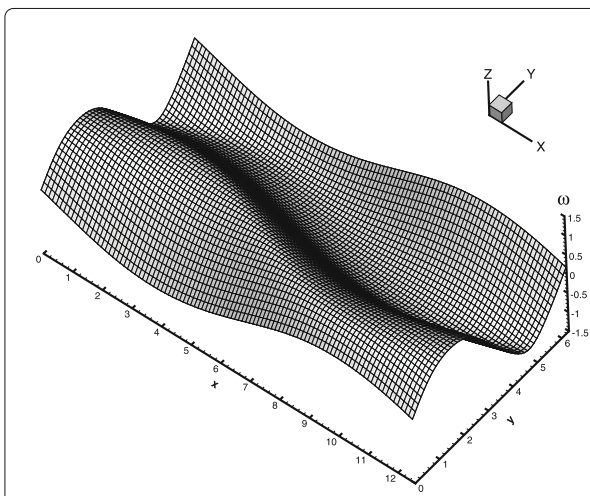

(a) Vorticity, $t=10$

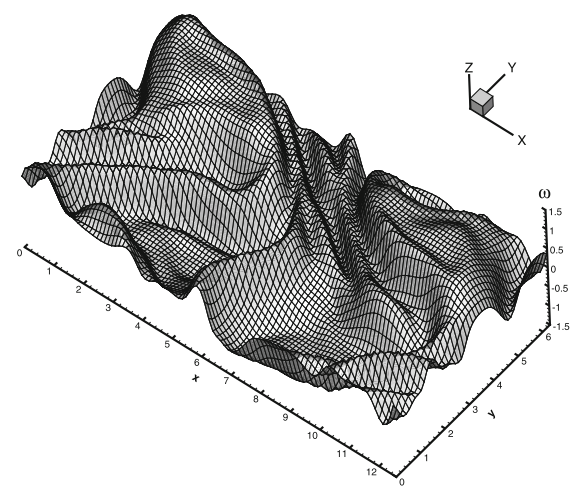

(c) Vorticity, $t=30$

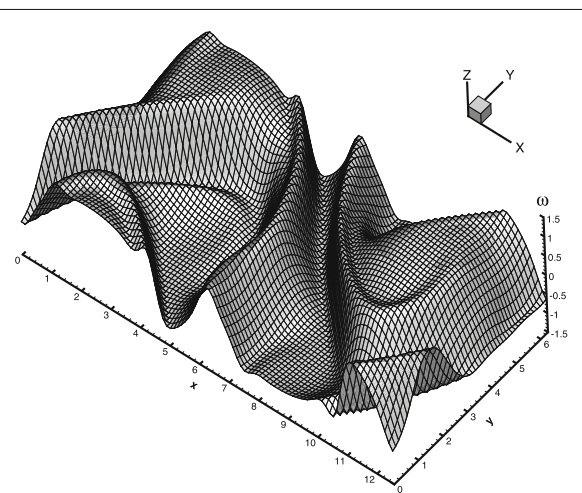

(b) Vorticity, $t=20$

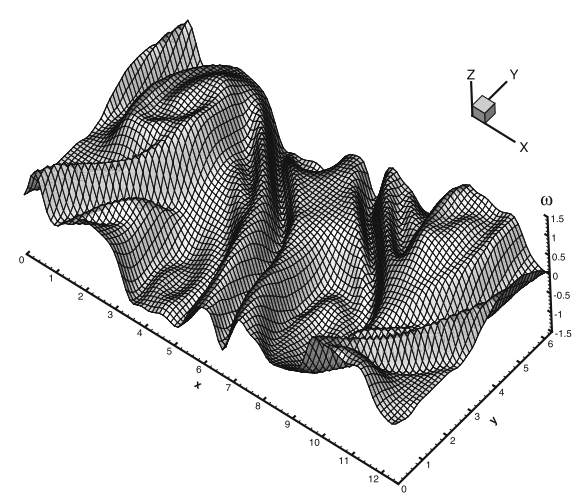

(d) Vorticity, $t=40$

Fig. 8 Example 8. Vorticity $\omega=v_{x}-u_{y}$ obtained with the GSA IMEX-ARS3 WCNS on a mesh $50 \times 50$ at different times. a Vorticity, $t=10$. b Vorticity, $t=20$. $\mathbf{c}$ Vorticity, $t=30$. d Vorticity, $t=40$

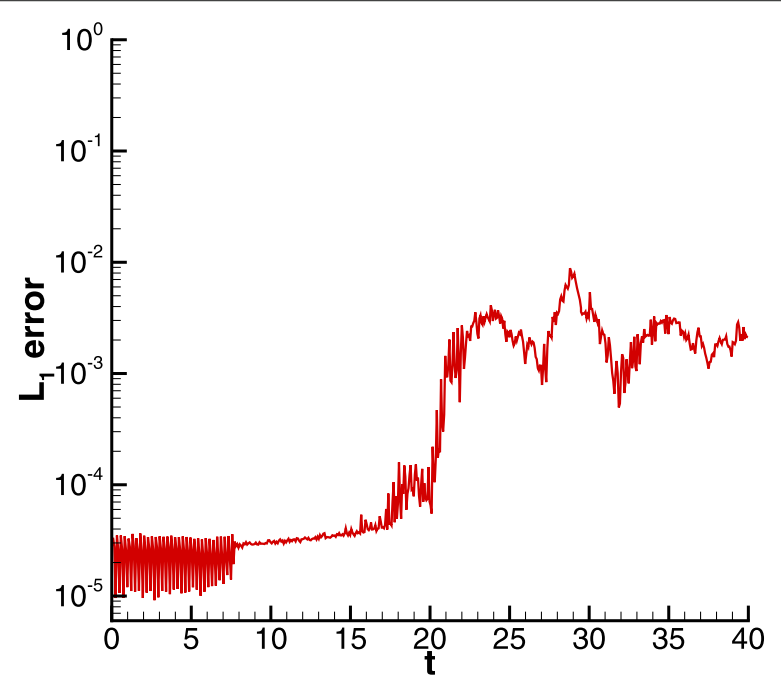

Fig. 9 Example 8. Time history of $L_{1}$ error of the divergence of the velocity $\nabla \cdot \mathbf{u}$ 


\section{Conclusions}

In this work, we have designed a high order IMEX WCNS for the compressibleincompressible limit problems described as scaled isentropic Euler equations. Based on the acoustic/advection splitting strategy, the semi-implicit method combines the thirdorder IMEX-ARS3 scheme for the time integration with the fifth-order WCNS for the spatial discretization. The GSA IMEX-ARS3 scheme has been proven to be asymptotic preserving, i.e., it converges to a consistent scheme in the zero Mach number limit, and asymptotically stable under a convective CFL condition. The IMEX-ARS3 WCNS has been verified numerically that it can achieve a third-order temporal accuracy for different values of the Mach number, from compressible to incompressible regimes. Numerical results show that the IMEX-ARS3 WCNS with the AP property can capture shocks and contact discontinuities with high resolution in the compressible regime, while it can capture incompressible features in the imcompressible regime. The future work will focus on the implementation of the proof of the AP property of the fully discrete scheme and the extension to compressible Navier-Stokes flows at any Mach number.

\section{Appendix}

This appendix is used to characterize the GSA and SA IMEX-ARSp schemes adopted in this paper.

The third-order GSA IMEX-RK3 scheme is characterized by

$$
\begin{aligned}
\mathbf{A} & =\left[\begin{array}{ccccc}
0 & 0 & 0 & 0 & 0 \\
0 & 0.5 & 0 & 0 & 0 \\
0 & 0.16666666666 & 0.5 & 0 & 0 \\
0 & -0.5 & 0.5 & 0.5 & 0 \\
0 & 1.5 & -1.5 & 0.5 & 0.5
\end{array}\right], \\
\tilde{\mathbf{A}} & =\left[\begin{array}{cccccc}
0 & 0 & 0 & 0 & 0 \\
0.5 & 0 & 0 & 0 & 0 \\
0.61111111111 & 0.0555555555 & 0 & 0 & 0 \\
0.8333333333 & -0.8333333333 & 0.5 & 0 & 0 \\
0.25 & 1.75 & 0.75 & -1.75 & 0
\end{array}\right],
\end{aligned}
$$

and $\mathbf{b}^{T}=(0,1.5,-1.5,0.5,0.5), \tilde{\mathbf{b}}^{T}=(0.25,1.75,0.75,-1.75,0)$. The third-order SA IMEX-RK3 scheme is characterized by

$$
\begin{aligned}
\mathbf{A} & =\left[\begin{array}{cccc}
0 & 0 & 0 & 0 \\
0 & 0.4358665215 & 0 & 0 \\
0 & 0.2820667392 & 0.4358665215 & 0 \\
0 & 1.2084966491 & -0.644363171 & 0.4358665215
\end{array}\right], \\
\tilde{\mathbf{A}} & =\left[\begin{array}{cccc}
0 & 0 & 0 & 0 \\
0.4358665215 & 0 & 0 & 0 \\
0.3212788860 & 0.3966543747 & 0 & 0 \\
-0.1058582961 & 0.5529291480 & 0.5529291480 & 0
\end{array}\right],
\end{aligned}
$$

and $\mathbf{b}^{T}=\tilde{\mathbf{b}}^{T}=(0,1.208496649,-0.644363171,0.4358665215)$. 
Newton-generalized minimum residual; MUSCL: Monotone upstream-centered scheme for conservation laws; TVD-RK3: Third-order total variation diminishing Runge-Kutta

\section{Acknowledgements}

Not applicable.

\section{Authors' contributions}

These authors contributed equally to this work. The author(s) read and approved the final manuscript.

\section{Funding}

The present study is supported by the National Numerical Wind Tunnel Project (No. NNW2018-ZT4A08), the National Natural Science Foundation of China (Nos. 11872323 and 11971025) and the Natural Science Foundation of Fujian Province (No. 2019J06002)

\section{Availability of data and materials}

All data generated or analysed during this study are included in this published article.

\section{Competing interests}

The authors declare that they have no competing interests.

\section{Author details}

'Department of Mathematics, Southwest University of Science and Technology, 621010 Mianyang, China. ${ }^{2}$ School of Mathematical Sciences, Fujian Provincial Key Laboratory of Mathematical Modeling and High-Performance Scientific Computing, Xiamen University, 361005 Xiamen, China. ${ }^{3}$ Computational Aerodynamics Institute, China Aerodynamics Research and Development Center, 621000 Mianyang, China.

Received: 20 July 2020 Accepted: 2 November 2020

Published online: 26 November 2020

\section{References}

1. Klainerman S, Majda A (1981) Singular limits of quasilinear hyperbolic systems with large parameters and the incompressible limit of compressible fluids. Commun Pure Appl Math 34:481-524

2. Klainerman S, Majda A (1982) Compressible and incompressible fluids. Commun Pure Appl Math 35:629-653

3. Boscarino S, Russo G, Scandurra L (2018) All Mach number second order semi-implicit scheme for the Euler equations of gas dynamics. J Sci Comput 77:850-884

4. Degond P, Tang M (2011) All speed scheme for the low Mach number limit of the isentropic Euler equations. Commun Comput Phys 10:1-31

5. Tang M (2012) Second order all speed method for the isentropic euler equations. Kinet Relat Models 5:155-184

6. Dimarco G, Vignal MH, Loubère R (2017) Study of a new asymptotic preserving scheme for the Euler system in the low Mach number limit. SIAM J Sci Comput 39:2099-2128

7. Li XS, Gu CW (2008) An all-speed roe-type scheme and its asymptotic analysis of low Mach number behaviour. J Comput Phys 227:5144-5159

8. Haack J, Jin S, Liu JG (2012) An all-speed asymptotic-preserving method for the isentropic Euler and Navier-Stokes equations. Commun Comput Phys 12:955-980

9. Cordier F, Degond P, Kumbaro A (2012) An asymptotic-preserving all speed scheme for the Euler and Navier-Stokes equations. J Comput Phys 231:5685-5704

10. Boscarino S, Qiu JM, Russo G, Xiong T (2019) A high order semi-implicit IMEX WENO scheme for the all-Mach isentropic Euler system. J Comput Phys 392:594-618

11. Goudon T, Llobell J, Minjeaud S (2020) An asymptotic preserving scheme on staggered grids for the barotropic Euler system in low Mach regimes. Numer Meth Part D E 36:1098-1128

12. Klein R (1995) Semi-implicit extension of a Godunov-type scheme based on low Mach number asymptotics I, One-dimensional flow. J Comput Phys 121:213-237

13. Noelle S, Bispen G, Arun KR, Lukacova-Medvidova M, Munz C-D (2014) A weakly asymptotic preserving low Mach number scheme for the Euler equations of gas dynamics. SIAM J Sci Comput 36:989-1024

14. Bispen G, Lukacova-Medvidova M, Yelash L (2017) Asymptotic preserving IMEX finite volume schemes for low Mach number Euler equations with gravitation. J Comput Phys 335:222-248

15. Zakerzadeh H, Noelle S (2018) A note on the stability of implicit-explicit flux-splittings for stiff systems of hyperbolic conservation laws. Commun Math Sci 16:1-15

16. Dimarco G, Loubere R, D. VM, Vignal MH (2018) Second order implicit-explicit total variation diminishing schemes for the Euler system in the low Mach regime. J Comput Phys 372:178-201

17. Jin S (1999) Efficient asymptotic-preserving (AP) schemes for some multiscale kinetic equations. SIAM I Sci Comput 21:441-454

18. Jin S (2012) Asymptotic preserving (AP) schemes for multiscale kinetic and hyperbolic equations: A review. Riv Mat Univ Parma 3:177-216

19. Arun KR, Samantaray S (2019) Asymptotic preserving and low Mach number accurate IMEX finite volume schemes for the Euler Equations. J Sci Comput 82:35-66

20. Tavelli M, Dumbser M (2017) A pressure-based semi-implicit space-time discontinuous Galerkin method on staggered unstructured meshes for the solution of the compressible Navier-Stokes equations at all Mach numbers. J Comput Phys 341:341-376

21. Deng XG, Zhang HX (2000) Developing high-order weighted compact nonlinear schemes. J Comput Phys 165:22-44 
22. Zhang SH, Jiang S, Shu C-W (2008) Development of nonlinear weighted compact schemes with increasingly higher order accuracy. J Comput Phys 227:7294-7321

23. Nonomura T, Fujii K (2013) Robust explicit formulation of weighted compact nonlinear scheme. Comput Fluids $85: 8-18$

24. Liu XL, Zhang SH, Zhang HX, Shu C-W (2015) A new class of central compact schemes with spectral-like resolution II: hybrid weighted nonlinear schemes. J Comput Phys 284:133-154

25. Deng XG, Mao ML, Jiang Y, Liu HY (2011) New high-order hybrid cell-edge and cell-node weighted compact nonlinear schemes. AIAA 2011-3857:1-10

26. Yan Z-G, Liu HY, Ma YK, Mao ML, Deng XG (2017) Further improvement of weighted compact nonlinear scheme using compact nonlinear interpolation. Comput Fluids 156:135-145

27. Wong ML, Lele SK (2017) High-order localized dissipation weighted compact nonlinear scheme for shock- and interface-capturing in compressible flows. J Comput Phys 339:179-209

28. Lele SK (1992) Compact finite difference schemes with spectral-like resolution. J Comput Phys 103:16-42

29. Deng XG, Jiang Y, Mao ML, Liu HY, Li S, Tu GH (2015) A family of hybrid cell-edge and cell-node dissipative compact schemes satisfying geometric conservation law. Comput Fluids 116:29-45

30. Jiang GS, Shu C-W (1996) Efficient implementation of weighted ENO schemes. J Comput Phys 126:202-228

31. Henrick AK, Aslam TD, Powers JM (2005) Mapped weighted essentially nonoscillatory schemes: achieving optimal order near critical points. J Comput Phys 207:542-567

32. Borges R, Carmona M, Costa B, Don WS (2008) An improved weighted essentially non-oscillatory scheme for hyperbolic conservation laws. J Comput Phys 227:3191-3211

33. Liu SP, Shen YQ, Zeng FJ, Yu M (2018) A new weighting method for improving the WENO-Z scheme. Int J Numer Meth Fluids 87:271-291

34. Luo X, Wu SP (2019) An improved fifth-order WENO-Z+ scheme. Chinese J Theor App Mech 51:1927-1939

35. Hu FX (2017) The weighted ENO scheme based on the modified smoothness indicator. Comput Fluids 150:1-7

36. Zheng SC, Deng XG, Wang DF, Xie CH (2019) A parameter-free $\epsilon$-adaptive algorithm for improving weighted compact nonlinear schemes. Int J Numer Meth Fluids 90:247-266

37. Ascher UM, Ruuth SJ, Spiteri RJ (1997) Implicit-explicit Runge-Kutta methods for time-dependent partial differential equations. Appl Numer Math 25:151-167

38. Kennedy CA, Carpenter MH (2003) Additive Runge-Kutta schemes for convection-diffusion-reaction equations. Appl Numer Math 44:139-181

39. Pareschi L, Russo G (2005) Implicit-explicit Runge-Kutta schemes and applications to hyperbolic systems with relaxation. J Sci Comput 25:129-155

40. Boscarino S (2007) Error analysis of IMEX Runge-Kutta methods derived from differential-algebraic systems. SIAM J Numer Anal 45:1600-1621

41. Boscarino S, Pareschi L, Russo G (2013) Implicit-explicit Runge-Kutta schemes for hyperbolic systems and kinetic equations in the diffusion limit. SIAM J Sci Comput 35:22-51

42. Kelley C (1995) Iterative methods for linear and nonlinear equations. Society for Industrial and Applied Mathematics, New Haven

43. Botti L (2015) A choice of forcing terms in inexact Newton iterations with application to pseudo-transient continuation for incompressible fluid flow computations. Appl Math Comput 266:713-737

44. van Leer B (1985) Upwind-difference methods for aerodynamic problems governed by the Euler equations. Lect Appl Math 22:327-335

\section{Publisher's Note}

Springer Nature remains neutral with regard to jurisdictional claims in published maps and institutional affiliations.

\section{Ready to submit your research? Choose BMC and benefit from:}

- fast, convenient online submission

- thorough peer review by experienced researchers in your field

- rapid publication on acceptance

- support for research data, including large and complex data types

- gold Open Access which fosters wider collaboration and increased citations

- maximum visibility for your research: over $100 \mathrm{M}$ website views per year

At $\mathrm{BMC}$, research is always in progress.

Learn more biomedcentral.com/submissions 